\title{
Efficient subdivision in hyperbolic groups and applications
}

\author{
Uri Bader, Alex Furman and Roman Sauer*
}

\begin{abstract}
We identify the images of the comparison maps from ordinary homology and Sobolev homology, respectively, to the $\ell^{1}$-homology of a word-hyperbolic group with coefficients in complete normed modules. The underlying idea is that there is a subdivision procedure for singular chains in negatively curved spaces that is much more efficient (in terms of the $\ell^{1}$ norm) than barycentric subdivision. The results of this paper are an important ingredient in a forthcoming proof of the authors that hyperbolic lattices in dimension $\geq 3$ are rigid with respect to integrable measure equivalence. Moreover, we prove a new proportionality principle for the simplicial volume of manifolds with word-hyperbolic fundamental groups.
\end{abstract}

Mathematics Subject Classification (2010). Primary 20F67; Secondary 55N99.

Keywords. Hyperbolic groups, measure equivalence, simplicial volume.

\section{Introduction and statement of the main results}

Bounded cohomology of (discrete or continuous) groups proved to be a useful tool for various questions about rigidity of groups. Since bounded cohomology is, in general, extremely hard to compute, the question of surjectivity or bijectivity of the comparison map from bounded cohomology to ordinary cohomology is very important. It is conjectured to be surjective (and might even be an isomorphism, for all we know) for simple connected Lie groups with finite center and trivial coefficients [3]. Furthermore, in degrees $\geq 2$ it is surjective for word-hyperbolic groups and arbitrary Banach modules as coefficients [19].

In this paper we are concerned with a kind of pre-dual situation: the comparison map from the ordinary homology of a discrete group into its $\ell^{1}$-homology. We prove in Theorem 1.5 that for word-hyperbolic groups the image of the comparison map in the $\ell^{1}$-homology coincides with the image of a similar comparison map from Sobolev homology (Definition 1.2) to the $\ell^{1}$-homology.

${ }^{*}$ U.B. and A.F. were supported in part by the BSF grant 2008267. U.B was supported in part by the ISF grant 704/08 and the GIF grant 2191-1826.6/2007. A.F. was supported in part by the NSF grants DMS 0604611 and 0905977 . R.S. gratefully acknowledges support from the Deutsche Forschungsgemeinschaft, made through grant SA 1661/1-2, during the initial phase of this project. 
The Sobolev chain complex $C_{*}^{(1,1)}(G)$ of a group $G$ can be viewed as a subcomplex of the $\ell^{1}$-chain complex $C_{*}^{(1)}(G)$, containing the ordinary chain complex $C_{*}(G)$, but being much larger than $C_{*}(G)$. The Sobolev homology of a group $G$ with coefficients in the Banach space $L^{1}(X)$, where $X$ is a $G$-probability space, is a natural recipient of certain maps associated to measure equivalence cocycles $G \times X \rightarrow G$ satisfying an $\ell^{1}$-condition. This is reminiscent of the work of Monod and Shalom [21], where bounded cohomology is used as a recipient of certain maps associated to measure equivalence cocycles, and where they prove rigidity results regarding measure equivalence of products of word-hyperbolic groups. In a forthcoming paper [1] we prove that hyperbolic lattices are rigid with respect to integrable measure equivalence using the main result of this paper, Theorem 1.5; in the present paper we give an application to the simplicial volume: in Theorem 1.9 we prove a proportionality principle with regard to integrable measure equivalence for manifolds with word-hyperbolic fundamental groups, which generalizes Gromov's proportionality principle [10], Section 0.4, for such manifolds.

1.1. Norms on the standard resolution and Sobolev homology. Let $X$ be a set. We consider the chain complex $C_{*}(X)$ where $C_{n}(X)$ is the free abelian group with basis $X^{n+1}$ and differentials defined by

$$
d_{n}\left(x_{0}, x_{1}, \ldots, x_{n}\right)=\sum_{i=0}^{n}(-1)^{i}\left(x_{0}, \ldots, \hat{x}_{i}, \ldots, x_{n}\right) .
$$

If $X=G$ is a group, then $C_{*}(G)$ is called the standard homogeneous resolution of $G$. Endowing each $C_{n}(G)$ with the diagonal $G$-operation, $C_{*}(G)$ becomes a chain complex of $\mathbb{Z} G$-modules. Let $C_{*}(G, \mathbb{R})=C_{*}(G) \otimes_{\mathbb{Z}} \mathbb{R}$ be the corresponding complex with real coefficients.

There is a variety of norms one might impose on $C_{*}(G)$; we consider the following:

Definition 1.1. Let $G$ be finitely generated. Fix a word metric on $G$. For a subset $S \subset G$ we denote by $\operatorname{diam}(S)$ the diameter with respect to this word metric. On $C_{n}(G)$ and $C_{n}(G, \mathbb{R})$ we define

(1) the $\ell^{1}$-norm

$$
\left\|\sum a_{\left(g_{0}, g_{1}, \ldots, g_{n}\right)} \cdot\left(g_{0}, g_{1}, \ldots, g_{n}\right)\right\|_{1}=\sum\left|a_{\left(g_{0}, g_{1}, \ldots, g_{n}\right)}\right|,
$$

and

(2) the Sobolev norm

$$
\begin{aligned}
\| \sum & a_{\left(g_{0}, g_{1}, \ldots, g_{n}\right)} \cdot\left(g_{0}, g_{1}, \ldots, g_{n}\right) \|_{1,1} \\
& =\sum\left|a_{\left(g_{0}, g_{1}, \ldots, g_{n}\right)}\right| \cdot\left(1+\operatorname{diam}\left(g_{0}, g_{1}, \ldots, g_{n}\right)\right) .
\end{aligned}
$$


It is easy to verify that different word metrics on $G$ give rise to equivalent Sobolev norms. We denote $C_{n}(G)$ when endowed with the Sobolev norm or $\ell^{1}$-norm by $C_{n}^{(1,1)}(G)$ and $C_{n}^{(1)}(G)$ respectively. Note that both are automatically complete because of the integral coefficients. The differentials above are continuous with respect to both the $\ell^{1}$-norm and the Sobolev norm. In particular, we obtain chain complexes of normed modules $C_{*}^{(1,1)}(G)$ and $C_{*}^{(1)}(G)$.

In Section 2 we explain the less common setting of normed rings and normed modules over normed rings. The integral group ring $\mathbb{Z} G$ endowed with the $\ell^{1}$-norm is a normed ring in the sense of definition 2.1. The chain complexes $C_{*}^{(1,1)}(G)$ and $C_{*}^{(1)}(G)$ are normed chain complexes over the normed ring $\mathbb{Z} G$. In subSection 2.2 we explain the construction of a completed tensor product $\hat{\otimes}_{\mathbb{Z} G}$ over the normed ring $\mathbb{Z} G$ endowed with the $\ell^{1}$-norm, which is an integral version of the projective tensor product of Banach spaces (compare Remark 2.11). It will be essential to the proof of Theorem 1.9 to use $\widehat{\otimes}_{\mathbb{Z} G}$ rather than the usual projective tensor product.

Definition 1.2. Let $E$ be a normed module $E$ over the normed ring $\mathbb{Z} G$. The $n$-th Sobolev homology $H_{n}^{(1,1)}(G, E)$ is the $n$-th homology of $C_{*}^{(1,1)}(G) \hat{\otimes}_{\mathbb{Z} G} E$. The $n$-th $\ell^{1}$-homology $H_{n}^{(1)}(G, E)$ is the $n$-th homology of $C_{*}^{(1)}(G) \hat{\otimes}_{\mathbb{Z} G} E$.

Definition 1.3. Let $E$ be a normed module $E$ over the normed ring $\mathbb{Z} G$. The homomorphisms $H_{*}(G, E) \rightarrow H_{*}^{(1)}(G, E)$ and $H_{*}^{(1,1)}(G, E) \rightarrow H_{*}^{(1)}(G, E)$ induced by the natural chain maps $C_{*}(G) \otimes_{\mathbb{Z} G} E \rightarrow C_{*}^{(1)}(G) \hat{\otimes}_{\mathbb{Z} G} E$ (compare Lemma 2.6) and $C_{*}^{(1,1)}(G) \hat{\otimes}_{\mathbb{Z} G} E \rightarrow C_{*}^{(1)}(G) \hat{\otimes}_{\mathbb{Z} G} E$ (compare Example 2.3), respectively, are called comparison maps.

Remark 1.4. If $E_{*}$ is a chain complex of normed modules over a normed ring, then its homology groups $H_{n}\left(E_{*}\right)$ inherit a semi-norm by defining the semi-norm of a homology class $x$ as the infimum of the norms of chains representing $x$.

Caveat. If $E=\mathbb{R}$ with the trivial $G$-action, then $H_{n}^{(1)}(G, E)$ is just the usual $\ell^{1}$ homology of $G$ as considered by various authors (see e.g. [17], [15]), i.e. the homology of the $\ell^{1}$-completion of the bar complex of $G$ with real coefficients (cf. Remark 2.11). The $\ell^{1}$-completion of the bar complex of $G$ is the pre-dual of the complex defining bounded cohomology of $G$.

We would like to warn the reader that in the literature (see e.g. the work of Mineyev [18]) the term $\ell^{1}$-homology is also used for the (usual) group homology of $G$ with coefficients in the $G$-module $\ell^{1}(G)$ which differs from our definition.

1.2. Main results. Our main theorem is:

Theorem 1.5. Let $G$ be a hyperbolic group. Let $E$ be a complete normed $\mathbb{Z} G$-module. Then the following images under the comparison maps coincide:

$$
\operatorname{im}\left(H_{*}(G, E) \rightarrow H_{*}^{(1)}(G, E)\right)=\operatorname{im}\left(H_{*}^{(1,1)}(G, E) \rightarrow H_{*}^{(1)}(G, E)\right) .
$$


The above theorem follows rather easily (see Section 3 ) from the following theorem. Here $C_{*}^{r}(G)$ denotes the Rips complex of $G$, which is a subcomplex of $C_{*}(G)$ (see Definition 3.1).

Theorem 1.6. Let $G$ be a $\delta$-hyperbolic group. There exist a $\mathbb{Z} G$-chain homomorphism $f_{*}: C_{*}(G) \rightarrow C_{*}(G)$ and constants $r(n)=r(n, \delta)>0$ for every $n \geq 0$ such that:

(1) $f_{0}=\mathrm{id}$,

(2) $\operatorname{im}\left(f_{i}\right) \subset C_{i}^{r(i)}(G)$ for $i \geq 0$, and

(3) $f_{i}$ is bounded with respect to the Sobolev norm on the domain and the $\ell^{1}$-norm on the target for $i \geq 0$.

Remark 1.7. The statement in the preceding theorem is actually true for some constant $r=r(i)$ that does not depend on $i$ (only on the group $G$ ), thus giving a chain map $f_{*}: C_{*}(G) \rightarrow C_{*}^{r}(G)$ which can be seen to be a homotopy equivalence. We refrain from a proof of this statement since it is more complicated, and the weaker statement in Theorem 1.6 is sufficient to conclude our main result, Theorem 1.5, and its corollaries presented below.

Remark 1.8. The map $f_{i}$ is a kind of subdivision map that maps arbitrarily large simplices in $C_{i}(G)$ to a sum of simplices of bounded diameter (bounded by $r(i)$ ). For $f_{i}$ to be continuous the number of simplices in this sum must grow at most linearly in the diameter of the large simplex you start with. That such an efficient subdivision is possible on trees is quite easy to see. We approximate simplices in $C_{i}(G)$ by trees (see Theorem 4.2) to reduce to this case.

Next we present an application of Theorem 1.5 to the simplicial volume of aspherical manifolds, which will be proved in Section 5. Recall that a topological space is aspherical if its universal cover is contractible. Two aspherical CW-complexes are homotopy equivalent if and only if their fundamental groups are isomorphic. The simplicial volume $\|M\| \in \mathbb{R} \geq 0$ of an $n$-dimensional closed orientable manifold $M$ is defined as the infimum of the $\ell^{1}$-norms of real singular chains representing the fundamental class in $H_{n}(M, \mathbb{R})$. The simplicial volume has many applications in geometry; see the groundbreaking paper of Gromov [10] for much more information.

The definitions of (integrable) measure equivalence and (integrable) ME-coupling will be recalled in Subsection 5.1. Measure equivalence is an equivalence relation between groups, introduced by Gromov in [12] as a measure-theoretic counter part to quasi-isometry between finitely generated groups; it is intimately related to orbit equivalence in ergodic theory, to the theory of von Neumann algebras, and to questions in descriptive set theory. We will not go further into a discussion of this notion here, but refer the reader to the surveys [24], [22], [8]. 
Theorem 1.9. ${ }^{1}$ Let $M$ and $N$ be closed, aspherical, orientable manifolds. Assume that there is an ergodic, integrable ME-coupling $(\Omega, \mu)$ of the fundamental groups $G=\pi_{1}(M)$ and $H=\pi_{1}(N)$ with coupling index $c_{\Omega}=\mu(H \backslash \Omega) / \mu(G \backslash \Omega)$. Then:

(1) If $\|N\|>0$ and $G$ is word-hyperbolic, then $\operatorname{dim}(N) \leq \operatorname{dim}(M)$.

(2) If $G$ and $H$ are word-hyperbolic and $M$ and $N$ have dimension $\geq 2$, then

$$
\|M\|=c_{\Omega} \cdot\|N\| \text { and } \operatorname{dim}(M)=\operatorname{dim}(N) .
$$

Since closed, aspherical, orientable manifolds with word-hyperbolic fundamental groups have positive simplicial volume (except in dimension 1) by Lemma 5.12, we obtain the following corollary.

Corollary 1.10. Let $M$ and $N$ be closed, aspherical, orientable manifolds of dimension at least 2 whose fundamental groups $G=\pi_{1}(M)$ and $H=\pi_{1}(N)$ are word-hyperbolic. Assume that there is an ergodic, integrable ME-coupling $(\Omega, \mu)$ of $G$ and $H$ with coupling index $c_{\Omega}=\mu(H \backslash \Omega) / \mu(G \backslash \Omega)$. Then $\|M\|=c_{\Omega} \cdot\|N\|$ and $\operatorname{dim}(M)=\operatorname{dim}(N)$. Further, if $H \cong G$, then $c_{\Omega}=1$.

Remark 1.11. Any ME-coupling $(\Omega, \mu)$ has a decomposition [7], Lemma 2.2, into ergodic ME-couplings $\left(\Omega, \mu_{z}\right)$. If $(\Omega, \mu)$ is integrable, then almost every $\left(\Omega, \mu_{z}\right)$ is integrable (see also [1]). Hence the equality of dimensions in the previous corollary also holds without the ergodicity assumption.

Remark 1.12. Let $M$ and $N$ be closed, orientable, negatively curved manifolds with isometric universal covers. Denote their universal cover by $W$. Then the isometry group of $W$ contains both $\pi_{1}(M)$ and $\pi_{1}(N)$ as cocompact lattices. In particular, the isometry group of $W$ endowed with the Haar measure is an integrable measure coupling. Up to the ergodicity assumption the previous corollary generalizes Gromov's proportionality principle [10], Section 0.4 , in that situation.

A positive answer to the following question would be a even more far-reaching generalization of the proportionality principle of the simplicial volume with strong consequences for the measure equivalence rigidity of hyperbolic lattices (see [1]); a positive answer would also fit well with the proportionality of $L^{2}$-Betti numbers with regard to measure equivalence [9] and a conjectural bound of $L^{2}$-Betti numbers by the simplicial volume [16], Conjecture 14.1 on p. 489.

Question 1.13. Let $M$ and $N$ be closed, orientable, aspherical manifolds. Assume that $\pi_{1}(M)$ and $\pi_{1}(N)$ are measure equivalent with index $c>0$. Does this imply that $\|M\|=c \cdot\|N\|$ ? Further, if both simplicial volumes are positive, are the dimensions of $M$ and $N$ equal?

\footnotetext{
${ }^{1}$ We thank Clara Löh for pointing out Lemma 5.12 to us, which allowed us to drop the assumption that the simplicial volumes are positive in assertion (2).
} 
1.3. Some global conventions. We use the terms hyperbolic group and word-hyperbolic group interchangeably. We also use the terms integrable ME-coupling and $\ell^{1}$ $M E$-coupling interchangeably. A $\delta$-hyperbolic group is understood in the sense of [2], Definition 1.1 on p. 399, using the slim triangles condition.

We denote the metric on a metric space generically by $d$; we also denote the differential in a chain complex generically by $d$, if it is clear without ambiguity.

\section{Normed rings and modules}

We transfer several concepts from topological vector spaces to the setting of $R$-modules, where $R$ is an arbitrary normed ring (for example, $R=\mathbb{Z}$ ). Most of this section is straightforward but we review basic notions like, e.g., completions and tensor products because it is not very common to consider normed modules over $\mathbb{Z}$ or $\mathbb{Z} G$.

Let $R$ be a unital ring, and let $1_{R}$ denote its unit element. We follow the usual convention and denote the element $n \cdot 1_{R}$ by $n \in R$.

Definition 2.1. Let $|\cdot|_{\mathbb{Z}}$ denote the usual absolute value on $\mathbb{Z}$. A normed ring $R$ is a unital ring $R$ endowed with a real-valued function $x \mapsto|x|_{R}$ (called norm) such that for all $x, y \in R$ and every $n \in \mathbb{Z}$ :

(1) $|x|_{R}=0 \Longleftrightarrow x=0$;

(2) $|x+y|_{R} \leq|x|_{R}+|y|_{R}$

(3) $|x y|_{R} \leq|x|_{R}|y|_{R}$;

(4) $|n x|_{R}=|n|_{\mathbb{Z}}|x|_{R}$.

Definition 2.2. A normed (left) $R$-module over a normed ring $R$ is a (left) $R$-module $M$ endowed with a real-valued norm function $m \mapsto\|m\|_{M}$ such that for all $u, v \in M$, $r \in R$, and $n \in \mathbb{Z}$ :

(1) $\|u\|_{M}=0 \Longleftrightarrow u=0$;

(2) $\|u+v\|_{M} \leq\|u\|_{M}+\|v\|_{M}$;

(3) $\|r u\|_{M} \leq|r|_{R}\|u\|_{M}$;

(4) $\|n u\|_{M}=|n|_{\mathbb{Z}}\|u\|_{M}$;

A normed right $R$-module is defined similarly.

If $\|\cdot\|$ on $M$ satisfies (2)-(4), but not necessarily (1), we call $M$ a semi-normed module. Whenever $M$ or $R$ are clear from the context, we denote the norms on $M$ or $R$ simply by $\|\cdot\|$ or $|\cdot|$, respectively. Observe that a normed module is necessarily torsion-free as an abelian group.

A normed complex over $R$ is just a chain complex in the category of normed $R$-modules. A bounded $R$-chain map is a chain map between normed complexes consisting of bounded $R$-homomorphisms in each degree. 
Example 2.3. Let $\mathbb{Z} G$ be endowed with the $\ell^{1}$-norm. Then $\mathbb{Z} G$ is a normed ring. The $\mathbb{Z} G$-modules $C_{k}(G)$ are normed modules when endowed with either the $\ell^{1}$ or the Sobolev norms (Definition 1.1). To emphasize the normed module structure on these modules we will denote them by $C_{k}^{(1)}(G)$ and $C_{k}^{(1,1)}(G)$, respectively. The boundary maps $d_{k}$ are bounded with respect to both norms (by $k+1$ ), thus both complexes $C_{*}^{(1)}(G)$ and $C_{*}^{(1,1)}(G)$ are normed $\mathbb{Z} G$-complexes. The inclusion $C_{*}^{(1,1)}(G) \hookrightarrow$ $C_{*}^{(1)}(G)$ is a bounded chain map of norm at most 1 .

2.1. Dual spaces and completions. Let $R$ be a normed ring. A homomorphism $\phi: M \rightarrow N$ between two normed $R$-modules $M, N$ is continuous with respect to the topologies induced by the norm of $M$ and $N$, respectively, if and only if it is bounded, that is, there is $c \geq 0$ such that $\|\phi(m)\| \leq c\|m\|$; the infimum of such constants $c$ is the operator norm $\|\phi\|$. In that case we say that $\phi$ is a bounded $R$-homomorphism.

Let $\operatorname{hom}_{R}^{\mathrm{b}}(M, N)$ be the abelian group of bounded $R$-homomorphisms from $M$ to $N$. Equipped with the operator norm it becomes a normed $\mathbb{Z}$-module. Every normed $R$-module has an underlying normed $\mathbb{Z}$-module. So we can define its dual

$$
M^{\prime}=\operatorname{hom}_{\mathbb{Z}}^{\mathrm{b}}(M, \mathbb{R}) .
$$

If $M$ is a normed left $R$-module, then $M^{\prime}$ is naturally a normed right $R$-module, and the double dual $M^{\prime \prime}$ is again a normed left $R$-module. In fact $M^{\prime}$ and $M^{\prime \prime}$ are real vector spaces (and modules over $\mathbb{R} \otimes_{\mathbb{Z}} R$ ).

Given a Cauchy sequence $\left(f_{i}\right)$ in $M^{\prime}$, one verifies that $f(m)=\lim _{i \rightarrow \infty} f_{i}(m)$ defines a bounded $f \in M^{\prime}$. Hence we obtain:

Lemma 2.4. $M^{\prime}$ is complete.

There is a natural map $M \rightarrow M^{\prime \prime}$, given, as usual, by an evaluation. The following is a version of the Hahn-Banach theorem that applies for normed modules.

Lemma 2.5 (Hahn-Banach for normed modules). Let $R$ be a normed ring, and let $M, N$ be semi-normed $R$-modules.

(1) For an injective bounded $R$-homomorphism $N \hookrightarrow M$, the induced dual map $N^{\prime} \rightarrow M^{\prime}$ is surjective.

(2) Every $m \in M$ has a supporting functional, that is,

$$
\forall m \in M \exists f \in M^{\prime} \text { such that }\|f\|=1 \text { and }\|f(m)\|=\|m\| .
$$

(3) The canonical bounded R-homomorphism $M \rightarrow M^{\prime \prime}$ given by evaluation is isometric. If $M$ is normed, it is also injective.

Proof. Firstly, since the $R$-linearity in the above statement is automatic, we regard $M, N$ as $\mathbb{Z}$-modules. Secondly, observe that it is enough to prove (1). Indeed, 
assertion (2) implies assertion (3), and assertion (1) implies (2) by setting $N=\mathbb{Z} m$, and letting $f$ be an extension of the map $N \rightarrow \mathbb{R}$ induced by $m \mapsto\|m\|$.

Assertion (1) can be easily reduced to the Hahn-Banach theorem for $\mathbb{Q}$-vector spaces and $\mathbb{R}$-valued functionals. Although the Hahn-Banach theorem for $\mathbb{Q}$-vector spaces is not commonly stated, the usual proof for real vector spaces (see e.g. [4], III §6) carries over verbatim.

The map $M \rightarrow \mathbb{Q} \otimes_{\mathbb{Z}} M, m \mapsto 1 \otimes m$ is an injection since $M$ is torsion-free. The following norm on $\mathbb{Q} \otimes_{\mathbb{Z}} M$ is the unique one that turns this injection into an isometry: Let $a_{i}, b_{i}$ integers for $i=1, \ldots, m$. Let $c=b_{1} b_{2} \cdots b_{n}$. Then we set

$$
\left\|\sum_{i=1}^{m} a_{i} / b_{i} \otimes m_{i}\right\|_{\mathbb{Q} \otimes_{\mathbb{Z}} M}=c^{-1}\left\|\sum_{i=1}^{m} \frac{a_{i} c}{b_{i}} m_{i}\right\|_{M} .
$$

The isometric embedding $M \hookrightarrow \mathbb{Q} \otimes_{\mathbb{Z}} M$ induces an isometric isomorphism $(\mathbb{Q} \otimes M)^{\prime} \rightarrow M^{\prime}$. Its inverse is given by

$$
M^{\prime} \ni f \mapsto \bar{f}, \quad \bar{f}(q \otimes m)=q f(m) .
$$

The proof of (1) now follows: for an injection of $\mathbb{Z}$-normed modules, $N \hookrightarrow M$, we obtain an injection $\mathbb{Q} \otimes N \hookrightarrow \mathbb{Q} \otimes M$ which induces by Hahn-Banach a surjection $(\mathbb{Q} \otimes M)^{\prime} \rightarrow(\mathbb{Q} \otimes N)^{\prime}$, thus a surjection $M^{\prime} \rightarrow N^{\prime}$.

The completion $\bar{M}$ of a (semi-)normed $R$-module $M$ is defined as the closure of the image of $M$ in $M^{\prime \prime}$. Using Lemma 2.5 (3) one easily sees that the completion satisfies the following universal property: Every bounded homomorphism of $M$ into a complete normed $R$-module $V$ extends uniquely to a bounded homomorphism from $\bar{M}$ to $V$.

2.2. Tensor products. Our next goal is to define the tensor product of normed modules. Our definition below is an extension of the construction known as the projective tensor product, which satisfies a universal property with respect to bilinear maps.

Given a normed right $R$-module $E$, a normed left $R$-module $F$, a normed $\mathbb{Z}$ module $V$ and a $\mathbb{Z}$-module morphism $\phi: E \otimes_{R} F \rightarrow V$, we obtain the associated $R$-bilinear map $\tilde{\phi}: E \times F \rightarrow V$. We set

$$
\|\tilde{\phi}\|=\inf \{c \geq 0 \mid\|\tilde{\phi}(f, e)\| \leq c\|f\|\|e\| \text { for all } f \in F, e \in E\},
$$

and say that $\tilde{\phi}$ is bounded if $\|\tilde{\phi}\|<\infty$.

Lemma 2.6. Let $E$ be a normed right $R$-module and $F$ be a normed left $R$-module. Then there is a complete normed $\mathbb{Z}$-module, denoted by $E \hat{\otimes}_{R} F$, and a $\mathbb{Z}$-homomor- 
phism $E \otimes_{R} F \stackrel{p}{\rightarrow} E \hat{\otimes}_{R} F$ satisfying the following universal property:

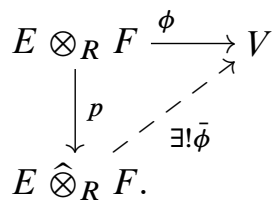

Expressed in words: For every complete $\mathbb{Z}$-module $V$ and for every $\mathbb{Z}$-homomorphism $\phi: E \otimes_{R} F \rightarrow V$ such that the associated bilinear map $\tilde{\phi}: E \times F \rightarrow V$ is bounded, there exist a unique bounded $\mathbb{Z}$-homomorphism $\bar{\phi}: E \hat{\otimes}_{R} F \rightarrow V$ such that $\phi=\bar{\phi} \circ p$ and $\|\bar{\phi}\|=\|\tilde{\phi}\|$.

Furthermore, the pair $\left(E \hat{\otimes}_{R} F, p\right)$ is unique up to isometric isomorphism.

Definition 2.7. Retain the setting of the previous lemma. The normed module $E \hat{\otimes}_{R} F$ is called the projective tensor product of $E$ and $F$ over $R$.

Proof. There is a natural $\mathbb{Z}$-module morphism:

$$
E \otimes_{R} F \rightarrow \operatorname{hom}_{R}^{\mathrm{b}}\left(E, F^{\prime}\right)^{\prime}, \quad e \otimes f \mapsto(T \mapsto\langle T e, f\rangle),
$$

where $\left\langle_{-},{ }_{-}\right\rangle$is the evaluation map $F^{\prime} \times F \rightarrow \mathbb{R}$. We denote the closure of the image by $E \hat{\otimes}_{R} F$ and the map $E \otimes_{R} \rightarrow E \hat{\otimes}_{R} F$ by $p$. By Lemma $2.4, \operatorname{hom}_{R}^{\mathrm{b}}\left(E, F^{\prime}\right)^{\prime}$ is complete, hence so is $E \hat{\otimes}_{R} F$.

For every complete $\mathbb{Z}$-module $V$ and for every $\mathbb{Z}$-homomorphism $\phi: E \otimes_{R} F \rightarrow$ $V$ such that the associated bilinear map $\tilde{\phi}: E \times F \rightarrow V$ is bounded, we obtain the map

$$
V^{\prime} \stackrel{\psi}{\rightarrow} \operatorname{hom}_{R}^{\mathrm{b}}\left(E, F^{\prime}\right), \quad \psi\left(v^{\prime}\right)=e \mapsto\left(f \mapsto v^{\prime}(\tilde{\phi}(e, f))\right),
$$

and it is clear that the composition

$$
E \otimes_{R} F \stackrel{p}{\rightarrow} \operatorname{hom}_{R}^{\mathrm{b}}\left(E, F^{\prime}\right)^{\prime} \stackrel{\psi^{\prime}}{\rightarrow} V^{\prime \prime}
$$

coincides with

$$
E \otimes_{R} F \stackrel{\phi}{\rightarrow} V \stackrel{i}{\rightarrow} V^{\prime \prime},
$$

where $i$ is the canonical map given by evaluation. Since $V$ is complete, $i(V)$ is closed in $V^{\prime \prime}$ by Lemma 2.5 , and therefore $\psi^{\prime-1}(i(V))$ is closed in $\operatorname{hom}_{R}^{\mathrm{b}}\left(E, F^{\prime}\right)^{\prime}$. It follows that the closure of $p\left(E \otimes_{R} F\right)$, that is $E \hat{\otimes}_{R} F$, lies in $\psi^{\prime-1}(i(V))$. Therefore $\psi^{\prime}\left(E \hat{\otimes}_{R} F\right) \subset i(V) \cong V$, and we obtain a map $\bar{\phi}: E \hat{\otimes}_{R} F \rightarrow V$ such that $\bar{\phi} \circ p=\phi$. We leave it to the reader to check using Lemma 2.5 that indeed $\|\bar{\phi}\|=\|\tilde{\phi}\|$.

The uniqueness of the pair ( $E \hat{\otimes}_{R} F, p$ ) up to isomorphism follows directly from the universal property. Observe that by choosing the above $\phi$ to be the identity map of $E \hat{\otimes}_{R} F$ we get that $\|\tilde{p}\|=1$. It follows that the unique isomorphism between modules having the above universal property is actually isometric. 
We summarize some of the properties of the projective tensor product. The proofs are easy and use the universal property above; we leave them to the reader.

Lemma 2.8. Let $M$ be an normed R-module. The following isomorphisms are natural and isometric:

(1) $R \hat{\otimes}_{R} M$ is isomorphic to the completion $\bar{M}$. In particular, $\mathbb{Z} \hat{\otimes}_{\mathbb{Z}} M$ is isomorphic to $\bar{M}$.

(2) $\mathbb{R} \hat{\otimes}_{\mathbb{Z}} M$ is a Banach space. If $M$ is a normed real vector space, $\mathbb{R} \hat{\otimes}_{\mathbb{Z}} M$ is isomorphic to $\bar{M}$. If $M$ is a Banach space, $\mathbb{R} \hat{\otimes}_{\mathbb{Z}} M$ is isomorphic to $M$.

(3) We have $M^{\prime} \cong\left(\mathbb{R} \widehat{\otimes}_{\mathbb{Z}} M\right)^{\prime} \cong \operatorname{hom}_{\mathbb{R}}^{\mathrm{b}}\left(\mathbb{R} \hat{\otimes}_{\mathbb{Z}} M, \mathbb{R}\right)$. In particular, if $M$ is a Banach space, then $M^{\prime}$ is isomorphic to the dual of $M$ as a Banach space.

The functor $M \mapsto \mathbb{R} \widehat{\otimes}_{\mathbb{Z}} M$ is called Banachification.

Example 2.9. Let $(X, \mu)$ be a measure space. Let $M$ be the abelian group consisting of finite-valued measurable functions from $X$ to $\mathbb{Z}$ supported on a set of finite measure. Integration gives a semi-norm on $M$, turning it into a semi-normed $\mathbb{Z}$-module. The completion $\bar{M}$ is denoted by $L^{1}(X, \mathbb{Z})$. It is the normed module consisting of $\mu$ integrable measurable maps $f: X \rightarrow \mathbb{Z}$ modulo null sets. The Banachification of $M$ is naturally identified with $L^{1}(X, \mathbb{R})$. The dual space $M^{\prime}$ is isomorphic to the Banach dual of $L^{1}(X, \mathbb{R})$, hence can be identified with $L^{\infty}(X, \mathbb{R})$.

Let $(Y, v)$ be another measure space. Let $\operatorname{pr}_{X}: X \times Y \rightarrow X$ and $\operatorname{pr}_{Y}: X \times Y \rightarrow Y$ be the projections onto $X$ and $Y$, respectively. The isometric bilinear map

$$
L^{1}(X, \mathbb{Z}) \times L^{1}(Y, \mathbb{Z}) \rightarrow L^{1}(X \times Y, \mathbb{Z}), \quad(f, g) \mapsto\left(f \circ \operatorname{pr}_{X}\right) \cdot\left(g \circ \operatorname{pr}_{Y}\right),
$$

where $X \times Y$ carries the product measure $\mu \times v$, induces, by the universal property, an isometric map

$$
L^{1}(X, \mathbb{Z}) \hat{\otimes}_{\mathbb{Z}} L^{1}(Y, \mathbb{Z}) \stackrel{\cong}{\rightrightarrows} L^{1}(X \times Y, \mathbb{Z}) .
$$

Since the image is closed and dense, this map is an isometric isomorphism. Similarly, we obtain an isometric isomorphism

$$
L^{1}(X, \mathbb{R}) \hat{\otimes}_{\mathbb{R}} L^{1}(Y, \mathbb{R}) \stackrel{\cong}{\cong} L^{1}(X \times Y, \mathbb{R}) .
$$

Example 2.10. Taking in the previous example $Y=G^{k+1}$ endowed with the counting measure, we obtain the isomorphisms

$$
\begin{aligned}
& C_{k}^{(1)}(G) \hat{\otimes}_{\mathbb{Z}} L^{1}(X, \mathbb{Z}) \stackrel{\cong}{\longrightarrow} L^{1}\left(G^{k+1} \times X, \mathbb{Z}\right), \\
& C_{k}^{(1)}(G) \hat{\otimes}_{\mathbb{Z}} L^{1}(X, \mathbb{R}) \stackrel{\cong}{\longrightarrow} L^{1}\left(G^{k+1} \times X, \mathbb{R}\right),
\end{aligned}
$$

where $G^{k+1} \times X$ carries the product of the counting measure and the given measure on $X$. 
If we endow $Y=G^{k+1}$ with the measure that assigns to each point $\left(g_{0}, \ldots, g_{k}\right)$ the weight $1+\operatorname{diam}\left(g_{0}, \ldots, g_{k}\right)$, then $L^{1}(Y) \cong C_{k}^{(1,1)}(G)$ as normed $\mathbb{Z} G$-modules, and we obtain an isomorphism

$$
\begin{aligned}
C_{k}^{(1,1)}(G) \hat{\otimes}_{\mathbb{Z}} L^{1}(X, \mathbb{Z}) \stackrel{\cong}{\cong}\left\{f \in L^{1}\left(G^{k+1} \times X, \mathbb{Z}\right) \mid\right. \\
\left.\int_{G^{k+1} \times X}\left|f\left(g_{0}, \ldots, g_{k}, x\right)\right|\left(1+\operatorname{diam}\left(g_{0}, \ldots, g_{k}\right)\right)<\infty\right\} ;
\end{aligned}
$$

similarly for $C_{k}^{(1,1)}(G) \hat{\otimes}_{\mathbb{Z}} L^{1}(X, \mathbb{R})$.

Remark 2.11. Let $E$ and $F$ be Banach spaces over $\mathbb{R}$. Observe that

$$
E \hat{\otimes}_{\mathbb{Z}} F \cong E \hat{\otimes}_{\mathbb{R}} F,
$$

since the left hand side satisfies the universal property of the right hand side. The universal property satisfied by $E \widehat{\otimes}_{\mathbb{R}} F$ is the one satisfied by the classical projective tensor product of Banach spaces. It follows that Definition 2.7 generalizes the classical definition of projective tensor product (see [13], [6]).

\section{Theorem 1.6 implies Theorem 1.5}

Throughout this section, let $G$ be a finitely generated group with a fixed word metric. We consider $\mathbb{Z} G$ as a normed ring endowed with the $\ell^{1}$ norm.

Definition 3.1 (Rips complex). Let $X$ be a metric space. Let $r>0$. We denote by $C_{*}^{r}(X)$ the subcomplex of $C_{*}(X)$ such that $C_{n}^{r}(X) \subset C_{n}(X)$ is the submodule generated by all $(n+1)$-tuples $\left(x_{0}, \ldots, x_{n}\right)$ whose diameter in $X$ is at most $r$. If $X=G$ is a group as above, then $C_{*}^{r}(G)$ is a $\mathbb{Z} G$-subcomplex of $C_{*}(G)$.

Theorem 3.2 ([2], 3.23 Proposition on p. 469). Let $G$ be a $\delta$-hyperbolic group. If $r \geq 4 \delta+6$, then

$$
H_{n}\left(C_{*}^{r}(G)\right)= \begin{cases}\mathbb{Z} & \text { if } n=0 \\ 0 & \text { if } n>0\end{cases}
$$

If we endow $C_{*}^{r}(G)$ with the $\ell^{1}$-norm, we use sometimes the notation $C_{*}^{r,(1)}(G)$.

Lemma 3.3. Let $E$ be a complete normed $\mathbb{Z} G$-module. For every $n \in \mathbb{N}$ the natural map

$$
C_{n}^{r}(G) \otimes_{\mathbb{Z} G} E \rightarrow C_{n}^{r,(1)}(G) \hat{\otimes}_{\mathbb{Z} G} E
$$

is an isomorphism. 
Proof. Let $N$ be the number of all tuples $\left(e, g_{1}, \ldots, g_{n}\right)$ in $G^{n+1}$ with diameter at most $r$. The $\mathbb{Z} G$-module $C_{n}^{r}(G)=C_{n}^{r,(1)}(G)$ is isomorphic to the free module $\mathbb{Z} G^{N}$. From that and from Lemma 2.8 one sees that both sides in (3.1) are canonically isomorphic to $E^{N}$.

We need the following continuous version of the fundamental lemma in homological algebra.

Lemma 3.4. Let $n \geq 1$. Let $\phi_{i}: C_{i}^{(1,1)}(G) \rightarrow C_{i}^{(1)}(G), 0 \leq i \leq n$, be a $\mathbb{Z} G$-chain homomorphism up to degree $n$, that is, we have $d \phi_{i}=\phi_{i-1} d$ for every $0 \leq i \leq n$. Assume that $\phi$ induces the identity on the zeroth homology. Then there are bounded $\mathbb{Z} G$-homomorphisms $h_{i}: C_{i}^{(1,1)}(G) \rightarrow C_{i+1}^{(1)}(G), 0 \leq i \leq n$, such that

$$
d h_{i}+h_{i-1} d=\phi_{i}-\mathrm{id} \text { for every } 0 \leq i \leq n,
$$

where $h_{-1} d=0$ is understood. That is, $h_{*}$ is a chain homotopy between $\phi_{*}$ and the identity up to degree $n$.

Proof. Recall that $C_{*}^{(1,1)}(G)=C_{*}(G)$ and $C_{*}^{(1)}(G)=C_{*}(G)$ as $\mathbb{Z} G$-modules. One verifies that $h_{i}^{\prime}: C_{i}(G) \rightarrow C_{i+1}(G)$ defined by

$$
h_{i}^{\prime}\left(g_{0}, \ldots, g_{i}\right)=\left(e, g_{0}, \ldots, g_{i}\right)
$$

is a (non-equivariant) chain contraction of the augmented chain complex $C_{*}(G)$ (see the comment on augmented chain complexes after Lemma 4.3).

Obviously, $h_{*}^{\prime}$ is continuous with respect to the $\ell^{1}$-norm. Let $x \in C_{1}(G)$ be an element such that $\phi_{0}(e)-e=d x$ for the unit $e \in G$; this element exists since $\phi_{*}$ induces the identity on 0-th homology. Then let $h_{0}: C_{0}(G) \rightarrow C_{1}(G)$ be the $\mathbb{Z} G$ homomorphism with $h_{0}(g)=g x$. Clearly, $h_{0}$ is bounded and satisfies $\phi_{0}-\mathrm{id}=d h_{0}$.

Now suppose that we have already constructed an equivariant bounded map $h_{i}: C_{i}(G) \rightarrow C_{i+1}(G)$ for $i=0, \ldots, k-1$, where $k \leq n$, such that

$$
d h_{i}+h_{i-1} d=\phi_{i}-\mathrm{id}
$$

for all $i=1, \ldots, k-1$ (where we set $\left.h_{-1}=0\right)$. Then define

$$
h_{k}\left(e, g_{1}, \ldots, g_{k}\right)=\left(h_{k}^{\prime} \circ\left(\phi_{k}-\mathrm{id}-h_{k-1} d\right)\right)\left(e, g_{1}, \ldots, g_{k}\right)
$$

and extend $h_{k}$ to all of $C_{k}(G)$ by $\mathbb{Z} G$-linearity. It is easy to see that $h_{k}$ is bounded with respect to the Sobolev norm in the domain and the $\ell^{1}$-norm in the target and satisfies (3.2).

Proof that Theorem 1.6 implies Theorem 1.5. Let $G$ be a $\delta$-hyperbolic group. Let $E$ be a complete normed $\mathbb{Z} G$-module. The $\subset$-inclusion in the statement of Theorem 1.5 is clear. Let $n \geq 0$. It remains to show that

$$
\operatorname{im}\left(H_{n}(G, E) \rightarrow H_{n}^{(1)}(G, E)\right) \supset \operatorname{im}\left(H_{n}^{(1,1)}(G, E) \rightarrow H_{n}^{(1)}(G, E)\right) .
$$


Let $r(i)=r(i, \delta), i \geq 0$, be the constants and $f_{*}: C_{*}(G) \rightarrow C_{*}(G)$ be the map provided by Theorem 1.6. Let

$$
r=\max \{4 \delta+6, r(0), r(1), \ldots, r(n+1)\} .
$$

The complex $C_{*}^{r}(G)$ is acyclic according to Theorem 3.2. We have $\operatorname{im}\left(f_{i}\right) \subset C_{i}^{r}(G)$ for every $0 \leq i \leq n+1$.

The map $f_{*}$ is a bounded chain homomorphism $f_{*}: C_{*}^{(1,1)}(G) \rightarrow C_{*}^{r,(1)}(G)$ up to degree $n+1$. Since $C_{*}^{r}(G)$ is acyclic, it is a free $\mathbb{Z} G$-resolution of $\mathbb{Z}$. Thus, by the fundamental lemma of homological algebra, there is a $\mathbb{Z} G$-chain map $g_{*}: C_{*}^{r}(G) \rightarrow$ $C_{*}(G)$. Since $C_{i}^{r}(G)$ is finitely generated as a $\mathbb{Z} G$-module for each $i \geq 0$, the map $g_{i}$ is automatically continuous with respect to the $\ell^{1}$-norms. Consider the following diagram for $* \leq n+1$ :

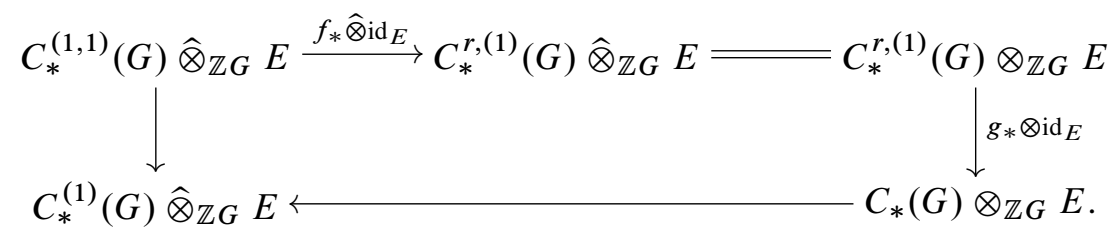

Note that $f_{*}$ induces a map $f_{*} \hat{\otimes} \operatorname{id}_{E}$ on the completed tensor products since it is continuous. The unlabeled arrows in the diagram are induced by natural inclusions. The equality in the diagram follows from Lemma 3.3. The diagram is commutative up to chain homotopy by Lemma 3.4. This implies (3.3).

\section{Tree approximation and the proof of Theorem 1.6}

4.1. Tree approximation. In this subsection we consider a version of Gromov's tree approximation of hyperbolic groups [11], Section 6, which is suited for our specific purposes.

Definition 4.1. Let $G$ be a finitely generated group. Fix a finite symmetric generating set in $G$. Let $\mathcal{G}$ be the corresponding Cayley graph of $G$. A family $\mathcal{W}=$ $\left\{w_{x, y}\right\}_{(x, y) \in G^{2}}$ is called a full family of geodesics in $G$ if $w_{x, y}:[0, d(x, y)] \rightarrow \mathscr{E}$ is a geodesic from $x$ to $y$ in $\mathscr{E}$ for every pair $(x, y) \in G^{2}$. For any $n$-tuple $Y=\left(y_{0}, \ldots, y_{n-1}\right) \in G^{n}$ let $[Y]_{w} \subset G$ be the set of vertices of the union $\mathcal{E}(Y)$ of the images of all geodesics $w_{y_{i}, y_{j}}$ with $i<j$.

Recall that a map between metric spaces $f: X \rightarrow Y$ is called a $c$-rough isometry if for every $x, x^{\prime} \in X$ we have

$$
\left|d\left(x, x^{\prime}\right)-d\left(f(x), f\left(x^{\prime}\right)\right)\right| \leq c \quad \text { for all } x, x^{\prime} \in X .
$$


The metric spaces $X$ and $Y$ are $c$-roughly isometric if there are $c$-rough isometries $f: X \rightarrow Y, g: Y \rightarrow X$ such that $d(x, g(f(x))) \leq c$ and $d(y, f(g(y))) \leq c$ for every $x \in X$ and every $y \in Y$. Furthermore, if there is a $c$-rough isometry $f: X \rightarrow Y$ such that $f(X)$ is $c$-dense in $Y$, then $X$ and $Y$ are $2 c$-roughly isometric.

A metric simplicial tree $T=(V, E)$ is a simplicial tree with vertices $V$ and edges $E$ endowed with a path metric $d$ on (the geometric realization of) $T$ such that each edge $e \in E$ is isometric to a compact interval $\left[0, l_{e}\right] \subset \mathbb{R}$.

Theorem 4.2. Let $G$ be a $\delta$-hyperbolic group. Let $\mathcal{W}=\left\{w_{x, y}\right\}_{(x, y) \in G^{2}}$ be a full family of geodesics in $G$. For every $n \in \mathbb{N}$ there is a constant $c=c(\delta, n)>0$ such that for every $n$-tuple $Y \in G^{n}$ the subspace $[Y] w$ is c-roughly isometric to a metric simplicial tree.

Proof. Consider an $n$-tuple $Y=\left(y_{0}, \ldots, y_{n-1}\right) \subset G$. Let $\mathcal{G}$ be the Cayley graph of $G$. Let

$$
\begin{aligned}
& \mathcal{E}(Y)_{0}=\bigcup_{0<i<n} \operatorname{im}\left(w_{y_{0}, y_{i}}\right), \\
& \mathscr{E}(Y)=\bigcup_{0 \leq i<j<n} \operatorname{im}\left(w_{y_{i}, y_{j}}\right) .
\end{aligned}
$$

Since the set of vertices in $\mathscr{Y}(Y)$, which is just $[Y]=[Y]$ w, is 1-dense, if $\mathscr{E}(Y)$ is $c$-roughly isometric to some metric space, then $[Y]$ is $(c+2)$-roughly isometric to the same space. We will now construct a rough isometry of $\mathscr{G}(Y)$ to a metric simplicial tree.

It is proved in [5], Théorème 1 on p. 91, that there is a constant $c^{\prime}(\delta, n)>0$, which only depends on $\delta$ and $n$, and a map $f: \mathscr{E}(Y)_{0} \rightarrow T$ to a metric simplicial tree $T$ such that for each $0<i<n$ the restriction $\left.\left.f\right|_{\operatorname{im}\left(w_{y_{0}}, y_{i}\right.}\right)$ is a bijective isometry and

$$
d(x, y)-c^{\prime}(\delta, n) \leq d(f(x), f(y)) \leq d(x, y)
$$

for all $x, y \in \mathscr{G}(Y)_{0}$. Note that $f$ is automatically surjective. The unique geodesic segment between points $z$ and $z^{\prime}$ of $T$ will be denoted by $\left[z, z^{\prime}\right] \subset T$. Next we extend $f$ to $\mathscr{G}(Y)$ as follows: For every $x \in \mathscr{G}(Y) \backslash \mathscr{E}(Y)_{0}$ choose $0<a(x) \leq n-1$ and $0<b(x) \leq n-1$ such that $x \in \operatorname{im}\left(w_{\left.y_{a(x)}, y_{b(x)}\right)}\right.$. Because of (4.1) we can pick a point $z \in\left[f\left(y_{a(x)}\right), f\left(y_{b(x)}\right)\right]$ such that

$$
\begin{aligned}
& \left|d\left(z, f\left(y_{a(x)}\right)\right)-d\left(x, y_{a(x)}\right)\right|<c^{\prime}(\delta, n), \\
& \left|d\left(z, f\left(y_{b(x)}\right)\right)-d\left(x, y_{b(x)}\right)\right|<c^{\prime}(\delta, n) .
\end{aligned}
$$

Then set $f(x)=z$.

To finish the proof, we show that $f: \mathscr{E}(Y) \rightarrow T$ satisfies

$$
\left|d\left(x, x^{\prime}\right)-d\left(f(x), f\left(x^{\prime}\right)\right)\right| \leq c \quad \text { for all } x, x^{\prime} \in \mathscr{E}(Y),
$$


where

$$
c=4 \delta+3 c^{\prime}(\delta, n) .
$$

Let $x$ and $x^{\prime}$ be points on the geodesics $w_{y_{i}, y_{j}}$ and $w_{y_{i^{\prime}}, y_{j^{\prime}}}$, where $i=a(x), j=b(x)$ and $i^{\prime}=a\left(x^{\prime}\right), j^{\prime}=b\left(x^{\prime}\right)$. By $\delta$-hyperbolicity there is a point $z$ on $w_{y_{0}, y_{i}}$ or $w_{y_{0}, y_{j}}$ such that

$$
d(x, z)<\delta .
$$

By the triangle inequality we obtain that

$$
\left|d\left(y_{i}, x\right)-d\left(y_{i}, z\right)\right|<\delta \text { and }\left|d\left(y_{j}, x\right)-d\left(y_{j}, z\right)\right|<\delta .
$$

Thus,

$$
\begin{aligned}
& \left|d\left(f\left(y_{i}\right), f(x)\right)-d\left(f\left(y_{i}\right), f(z)\right)\right|<\delta+c^{\prime}(\delta, n), \\
& \left|d\left(f\left(y_{j}\right), f(x)\right)-d\left(f\left(y_{j}\right), f(z)\right)\right|<\delta+c^{\prime}(\delta, n) .
\end{aligned}
$$

Since $T$ is tree and $f(x) \in\left[f\left(y_{i}\right), f\left(y_{j}\right)\right]$, either $f(x) \in\left[f\left(y_{i}\right), f(z)\right]$, or $f(x) \in$ $\left[f(z), f\left(y_{j}\right)\right]$. In both cases (4.4) implies that

$$
d(f(x), f(z))<\delta+c^{\prime}(\delta, n) .
$$

Similarly, we find a point $z^{\prime}$ on $w_{y_{0}, y_{i^{\prime}}}$ or $w_{y_{0}, y_{j^{\prime}}}$ such that

$$
d\left(x^{\prime}, z^{\prime}\right)<\delta \quad \text { and } \quad d\left(f\left(x^{\prime}\right), f\left(z^{\prime}\right)\right)<\delta+c^{\prime}(\delta, n) .
$$

From (4.5), (4.6), (4.3) and the fact that $f$ is a $c^{\prime}(\delta, n)$-rough isometry on $\mathcal{E}(Y)_{0}$ we obtain that

$$
\begin{aligned}
d\left(x, x^{\prime}\right) \leq d\left(z, z^{\prime}\right)+2 \delta & \leq d\left(f(z), f\left(z^{\prime}\right)\right)+2 \delta+c^{\prime}(\delta, n) \\
& \leq d\left(f(x), f\left(x^{\prime}\right)\right)+4 \delta+3 c^{\prime}(\delta, n) .
\end{aligned}
$$

Similarly, we get

$$
d\left(x, x^{\prime}\right) \geq d\left(f(x), f\left(x^{\prime}\right)\right)+4 \delta+3 c^{\prime}(\delta, n),
$$

which proves (4.2).

\subsection{An efficient chain contraction of the Rips complex to a tree}

Lemma 4.3. For every $r \geq 1$ and every $n \in \mathbb{N}$ there is a constant $e(r, n)>0$ with the following property: Let $T$ be a metric simplicial tree. Let $V$ be a subset of the vertices of $T$ such that the distance of any two distinct vertices in $V$ is at least 1 . Then there is a chain contraction $h_{i}^{T}: C_{i}^{r}(V) \rightarrow C_{i+1}^{r}(V), i \geq-1$, of the augmented chain complex $C_{*}^{r}(V)$ such that

$$
\left\|h_{i}^{T}\right\|<e(r, i) \text { for every } i \geq 1,
$$

where the operator norm is taken with respect to the $\ell^{1}$-norms. 
Here we mean by the augmented chain complex the complex $C_{*}^{r}(V)$ extended by $C_{-1}^{r}(V)=\mathbb{Z}$ and the differential (augmentation) $d: C_{0}^{r}(V) \rightarrow \mathbb{Z}$ that maps every $v \in V$ to $1 \in \mathbb{Z}$.

Proof. Fix a base point $x \in V$. Let $h_{-1}^{T}$ be defined by $h_{-1}^{T}(1)=x$. For every $v \in V$ we define $h_{0}^{T}$ by

$$
h_{0}^{T}(v)=\sum_{i=0}^{m-1}\left(v_{i}, v_{i+1}\right),
$$

where $x=v_{0}, v_{1}, \ldots, v_{m}=v$ (in that order) are the vertices in $V$ lying on the unique geodesic from $x$ to $v$. It is clear that

$$
d h_{0}^{T}(v)=v-x=\left(\mathrm{id}-h_{-1}^{T} d\right)(x) .
$$

For $v \in V$ and $i \geq 0$ consider the linear map given by

$$
c_{v}: C_{i}(V) \rightarrow C_{i+1}(V), \quad c_{v}\left(v_{0}, \ldots, v_{i}\right)=\left(v, v_{0}, \ldots, v_{i}\right) .
$$

One verifies that for $i \geq 1, v \in V$, and $\left(v_{0}, \ldots, v_{i}\right) \in V^{i+1}$ we have

$$
d c_{v}\left(v_{0}, \ldots, v_{i}\right)=\left(\mathrm{id}-c_{v} d\right)\left(v_{0}, \ldots, v_{i}\right) .
$$

We define the homomorphisms $h_{i}^{T}: C_{i}^{r}(V) \rightarrow C_{i+1}(V)$ for $i=1,2, \ldots$ inductively by

$$
h_{i}^{T}\left(v_{0}, \ldots, v_{i}\right)=c_{v_{0}}\left(\left(\mathrm{id}-h_{i-1}^{T} d\right)\left(v_{0}, \ldots, v_{i}\right)\right) .
$$

It follows inductively from the following computation that $h_{*}^{T}$ is a chain contraction:

$$
\begin{aligned}
d h_{i+1}^{T}\left(v_{0}, \ldots, v_{i+1}\right) & =d c_{v_{0}}\left(\mathrm{id}-h_{i}^{T} d\right)\left(v_{0}, \ldots, v_{i+1}\right) \\
& =\left(\mathrm{id}-c_{v_{0}} d\right)\left(\mathrm{id}-h_{i}^{T} d\right)\left(v_{0}, \ldots, v_{i+1}\right) \\
& =\left(\mathrm{id}-h_{i}^{T} d-c_{v_{0}} d+c_{v_{0}} d h_{i}^{T} d\right)\left(v_{0}, \ldots, v_{i+1}\right) \\
& =\left(\mathrm{id}-h_{i}^{T} d-c_{v_{0}} d+c_{v_{0}}\left(\mathrm{id}-h_{i-1}^{T} d\right) d\right)\left(v_{0}, \ldots, v_{i+1}\right) \\
& =\left(\mathrm{id}-h_{i}^{T} d\right)\left(v_{0}, \ldots, v_{i+1}\right) .
\end{aligned}
$$

Next we define $e(r, i)$ and show that (4.7) holds by induction. Set $e(r, 1)=r+1$. Let $(u, v) \in C_{1}^{r}(V)$ be a 1 -simplex. Let $v=z_{0}, z_{1}, \ldots, z_{m}=u$ (in that order) be the vertices in $V$ lying on the unique geodesic from $v$ to $u$. Since $T$ is a tree, we get that

$$
h_{0}^{T} d(u, v)=\sum_{k=0}^{m-1}\left(z_{k}, z_{k+1}\right) .
$$

Since the distance from $u$ to $v$ is $\leq r$ and the distance from $z_{k}$ to $z_{k+1}$ is $\geq 1$ by assumption, we have $m \leq r$ and thus $\left\|h_{0} d(u, v)\right\|_{1} \leq r$. This implies $\left\|h_{1}\right\| \leq e(r, 1)$. For $i \geq 2$ set

$$
e(r, i)=e(r, i-1) \cdot(i+1)+1 .
$$


Because of $e(r, 1)=r+1$ one sees that $e(r, i)$ only depends on $r$ and $i$, but not on the specific tree $T$. Definition (4.8) and the fact that the differential in degree $i$ has norm at most $i+1$ yield (4.7).

Finally we prove that $\operatorname{im}\left(h_{i}^{T}\right) \subset C_{i+1}^{r}(V)$. It suffices to show that for every $i \geq 1$ and every $\left(v_{0}, \ldots, v_{i}\right) \in C_{i}^{r}(V)$ we have

$$
\operatorname{supp}\left(h_{i}^{T}\left(v_{0}, \ldots, v_{i}\right)\right) \subset \operatorname{conv}\left(v_{0}, \ldots, v_{i}\right) .
$$

Here the support $\operatorname{supp}(s)$ of an element $s \in C_{i}(V)$, which can be uniquely written as a linear combination of $(i+1)$-tuples in $V^{i+1}$, is the union of all $v \in V$ that appear in one of these $(i+1)$-tuples. We denote the convex hull of a set $S \subset V$ by $\operatorname{conv}(S)$. We have (4.10) for $i=1$ by definition (4.8) and because all the points $z_{i}$ in (4.9) lie on the geodesic from $u$ to $v$. If (4.10) holds for $h_{i}^{T}$ with $i \geq 1$, it is true for $h_{i+1}^{T}$ because of

$$
\begin{aligned}
\operatorname{supp}\left(h_{i+1}^{T}\left(v_{0}, \ldots, v_{i+1}\right)\right) & \subset\left\{v_{0}, \ldots, v_{i+1}\right\} \cup \bigcup_{k=0}^{i+1} \operatorname{supp}\left(h_{i}^{T}\left(v_{0}, \ldots, \widehat{v}_{k}, \ldots, v_{i+1}\right)\right) \\
& \subset\left\{v_{0}, \ldots, v_{i+1}\right\} \cup \bigcup_{k=0}^{i+1} \operatorname{conv}\left(v_{0}, \ldots, \widehat{v}_{k}, \ldots, v_{i+1}\right) \\
& =\operatorname{conv}\left(v_{0}, \ldots, v_{i+1}\right) .
\end{aligned}
$$

\subsection{Proof of Theorem 1.6}

Proof. Choose a full family $\mathcal{W}=\left\{w_{x, y}\right\}_{(x, y) \in G^{2}}$ of geodesics in $G$ that is $G$-equivariant in the sense that for all $x, y, g \in G$ we have $g w_{x, y}=w_{g x, g y}$.

For a $k$-tuple $Y \in G^{k}$ we write $[Y]$ instead of $[Y]_{w}$ in the sequel. For $i=0,1, \ldots$ we define inductively real numbers $r(i) \geq 1$ and $\mathbb{Z} G$-homomorphisms $f_{i}: C_{i}(G) \rightarrow$ $C_{i}^{r(i)}(G)$ for $i \in \mathbb{N}$ such that

a) $f_{0}$ is the identity,

b) $d f_{i}=f_{i-1} d$,

c) $f_{i}$ is bounded when endowing the source with the Sobolev and the target with the $\ell^{1}$ norm, and

d) for every $\left(g_{0}, \ldots, g_{i}\right) \in C_{i}(G)$ we have

$$
f_{i}\left(\left(g_{0}, \ldots, g_{i}\right)\right) \in C_{i}^{r(i)}\left(\left[\left(g_{0}, \ldots, g_{i}\right)\right]\right) .
$$

The theorem follows from a)-c). Property d) is just needed for running the induction argument.

The basis of the induction will be an explicit construction of $f_{0}$ and $f_{1}$. We set $r(0)=r(1)=1$. Define $f_{0}$ to be the identity map. If for $x, y \in G$ the points 
$x=z_{0}, z_{1}, \ldots, z_{d}=y$ are the successive vertices on the geodesic $w_{x, y}$ from $x$ to $y$, we define $f_{1}$ by

$$
f_{1}((x, y))= \begin{cases}\sum_{i=1}^{d}\left(z_{i-1}, z_{i}\right) & \text { if } i \geq 1 \\ (x, y) & \text { if } i=0 \text { and } x=y .\end{cases}
$$

It is clear that $f_{0}$ and $f_{1}$ respect all the properties above.

Fix $i \geq 1$ and assume $f_{j}$ is already defined for $0 \leq j \leq i$ satisfying a)-d). According to Theorem 4.2 there is a constant $c(i)>0$ such that for every $Y \in G^{i+1}$ the subspace $[Y]$ is $c(i)$-roughly isometric to metric simplicial tree. We set

$$
r(i+1)=r(i)+2 c(i) .
$$

By Lemma 4.3 for every $n \in \mathbb{N}$ there is a constant $e(i, n)>0$ such that for every metric simplicial tree $T$ with a subset $V$ of vertices whose distinct elements have distance $\geq 1$ from each other there is a chain contraction

$$
h_{*}^{T}: C_{*}^{r(i)+c(i)}(V) \rightarrow C_{*+1}^{r(i)+c(i)}(V)
$$

of the augmented chain complex such that the operator norm with respect to the $\ell^{1}$-norm satisfies $\left\|h_{n}\right\|<e(i, n)$. Let

$$
B=\left\{\left(e, g_{1}, \ldots, g_{i+1}\right) ; g_{k} \in G \text { for } 1 \leq k \leq i+1\right\} .
$$

Note that $B$ is $\mathbb{Z} G$-basis of $C_{i+1}(G)$. After some preparation we define $f_{i+1}(\sigma) \in$ $C_{i+1}^{r(i+1)}(G)$ in (4.11) for every $\sigma \in B$ such that

e) $d f_{i+1}(\sigma)=f_{i} d(\sigma)$

f) $\left\|f_{i+1}(\sigma)\right\|_{1} \leq(e(i, i+1)+(i+1))\left\|f_{i}\right\|(i+2)\|\sigma\|_{1,1}$, and

g) $f_{i+1}(\sigma) \in C_{i+1}^{r(i+1)}([\sigma])$

hold for every $\sigma \in B$. The theorem then follows from the following easy claim, which we leave to the reader.

Claim. The $\mathbb{Z} G$-linear extension to $C_{i+1}(G) \rightarrow C_{i+1}^{r(i+1)}(G)$ of a map $f_{i+1}: B \rightarrow$ $C_{i+1}^{r(i+1)}(G)$ satisfying e) $-\mathrm{g}$ ) satisfies b)-d). The extension

$$
f_{i+1}: C_{i+1}(G) \rightarrow C_{i+1}^{r(i+1)}(G)
$$

has operator norm

$$
\left\|f_{i+1}\right\| \leq(e(i, i+1)+(i+1))\left\|f_{i}\right\|(i+2) .
$$

Let $\sigma \in B$. Let $T^{\sigma}$ be a metric simplicial tree such that $[\sigma]$ is $c(i)$-roughly isometric to $T^{\sigma}$. Let $V^{\sigma}$ be a set of points of $T^{\sigma}$ such that any two distinct points in $V^{\sigma}$ have distance $\geq 1$ and $V^{\sigma}$ is 3-dense in $T^{\sigma}$. By subdividing $T^{\sigma}$ we may assume 
that $V^{\sigma}$ consists of vertices. Upon increasing $c(i)$ by $6=2 \cdot 3$, thus $r(i+1)$ by $12=2 \cdot 6$, we may and will assume that $[\sigma]$ is also $c(i)$-roughly isometric to $V^{\sigma}$. Let

$$
\phi^{\sigma}:[\sigma] \rightarrow V^{\sigma} \quad \text { and } \quad \psi^{\sigma}: V^{\sigma} \rightarrow[\sigma]
$$

be two $c(i)$-rough isometries such that

$$
d\left(\phi^{\sigma} \psi^{\sigma}, \operatorname{id}_{V^{\sigma}}\right) \leq c(i) \text { and } d\left(\psi^{\sigma} \phi^{\sigma}, \operatorname{id}_{[\sigma]}\right) \leq c(i) .
$$

The maps $\phi^{\sigma}$ and $\psi^{\sigma}$ induce chain maps

$$
\phi_{*}^{\sigma}: C_{*}^{r(i)}([\sigma]) \rightarrow C_{*}^{r(i)+c(i)}\left(V^{\sigma}\right) \quad \text { and } \quad \psi_{*}^{\sigma}: C_{*}^{r(i)+c(i)}\left(V^{\sigma}\right) \rightarrow C_{*}^{r(i+1)}([\sigma]) .
$$

The next claim follows by a straightforward computation.

Claim. Let $r>0$. The map $h_{*}^{\sigma}: C_{*}^{r(i)}([\sigma]) \rightarrow C_{*+1}^{r(i+1)}([\sigma])$ defined by

$$
h_{n}^{\sigma}\left(g_{0}, \ldots, g_{n}\right)=\sum_{k=0}^{n}(-1)^{k}\left(g_{0}, \ldots, g_{k}, \psi^{\sigma} \phi^{\sigma}\left(g_{k}\right), \ldots, \psi^{\sigma} \phi^{\sigma}\left(g_{n}\right)\right)
$$

is a chain homotopy between the composition $\psi_{*}^{\sigma} \phi_{*}^{\sigma}: C_{*}^{r(i)}([\sigma]) \rightarrow C_{*}^{r(i+1)}([\sigma])$ and the identity, that is, $\psi_{n}^{\sigma} \phi_{n}^{\sigma}-\mathrm{id}=d h_{n}^{\sigma}+h_{n-1}^{\sigma} d$ for every $n \geq 0$, where we set $h_{-1}^{\sigma}=0$.

For $\sigma \in B$ define now

$$
f_{i+1}(\sigma)=\psi_{i+1}^{\sigma} h_{i}^{T_{\sigma}} \phi_{i}^{\sigma} f_{i} d(\sigma)-h_{i}^{\sigma} f_{i} d(\sigma) \in C_{i+1}^{r(i+1)}([\sigma]) .
$$

Property g) is clear from the definitions. The differential in degree $(i+1)$ of $C_{*}(G)$ (endowed with the Sobolev norm) has norm at most $(i+2)$, and $h_{i}^{\sigma}$ has norm at most $(i+1)$ (with respect to the $\ell^{1}$-norms). The maps $\phi_{i}^{\sigma}$ and $\psi_{i}^{\sigma}$ have norm at most 1 . Hence we obtain that

$$
\left\|f_{i+1}(\sigma)\right\|_{1} \leq(e(i, i+1)+(i+1))\left\|f_{i}\right\|(i+2)\|\sigma\|_{1,1} .
$$

Property e) follows from

$$
\begin{aligned}
d f_{i+1}(\sigma) & =d\left(\psi_{i+1}^{\sigma} h_{i}^{T_{\sigma}} \phi_{i}^{\sigma} f_{i} d-h_{i}^{\sigma} f_{i} d\right)(\sigma) \\
& =\left(\psi_{i}^{\sigma} d h_{i}^{T_{\sigma}} \phi_{i}^{\sigma} f_{i} d-d h_{i}^{\sigma} f_{i} d\right)(\sigma) \\
& =\left(\psi_{i}^{\sigma}\left(\mathrm{id}-h_{i-1}^{T_{\sigma}} d\right) \phi_{i}^{\sigma} f_{i} d-\left(\psi_{i}^{\sigma} \phi_{i}^{\sigma}-\mathrm{id}-h_{i-1}^{\sigma} d\right) f_{i} d\right)(\sigma) \\
& =\left(f_{i} d-\psi_{i}^{\sigma} h_{i-1}^{T_{\sigma}} d \phi_{i}^{\sigma} f_{i} d\right)(\sigma)+\left(h_{i-1}^{\sigma} f_{i-1} d d\right)(\sigma) \\
& =\left(f_{i} d-\psi_{i}^{\sigma} h_{i-1}^{T_{\sigma}} \phi_{i-1}^{\sigma} f_{i-1} d d\right)(\sigma) \\
& =f_{i} d(\sigma)
\end{aligned}
$$




\section{Integrable measure equivalence and simplicial volume}

5.1. Integrable measure equivalence. We recall the central notion of measure equivalence which was suggested by Gromov [12], 0.5.E.

Definition 5.1. Two countable groups $G, H$ are called measure equivalent if there is a standard measure space $(\Omega, \mu)$ with commuting $\mu$-preserving $G$ - and $H$-actions, such that each one of the actions admits a finite measure fundamental domain. The space $(\Omega, \mu)$ endowed with these actions is called an $M E$-coupling of $G$ and $H$.

Given measure equivalent groups $G$ and $H$, an actual choice of fundamental domains is not a part of the structure of an ME-coupling of $G$ and $H$. But it is easy to see that the measures of $G$ - and $H$-fundamental domains are independent of the choice. So for an ME-coupling $(\Omega, \mu)$ of $G$ and $H$, the ratio $c_{\Omega}=\mu\left(X_{H}\right) / \mu\left(X_{G}\right)$ of the measure of an $H$-fundamental domain by the measure of a $G$-fundamental domain is well defined and called the coupling index of $\Omega$.

The map $X_{G} \hookrightarrow \Omega \rightarrow G \backslash \Omega$ is a measure isomorphism. Since $H$ acts on $G \backslash \Omega$, this identification induces a measurable action of $H$ on $X_{G}$, for which we use the dot notation $h \cdot x$ for $h \in H$ and $x \in X_{H}$ to distinguish it from the action $h x$ of $H$ on $\Omega$. Similarly for $X_{H}$.

The coupling $\Omega$ is called ergodic if the $H$-action on $G \backslash \Omega$ is ergodic, or equivalently, the $G$-action on $H \backslash \Omega$ is ergodic [7], Lemma 2.2.

Definition 5.2. Let $(\Omega, \mu)$ be an ME-coupling of $G$ and $H$. Let $X_{G} \subset \Omega$ and $X_{H} \subset \Omega$ be fundamental domains of the $G$ - and $H$-action, respectively.

(1) We define $\alpha_{X_{H}}$ as

$$
\alpha_{X_{H}}: G \times X_{H} \rightarrow H, \quad(g, x) \mapsto h \quad \text { with } g x \in h^{-1} X_{H},
$$

and call $\alpha_{X_{H}}$ the (measurable) cocycle associated to $X_{H}$. Similarly for $\alpha_{X_{G}}$.

(2) Assume that $H$ is finitely generated, and let $l: H \rightarrow \mathbb{N}$ be the length function associated to some word-metric on $H$. We say that the fundamental domain $X_{H}$ is integrable if the function $x \mapsto l\left(\alpha_{X_{H}}(g, x)\right)$ is in $L^{1}\left(X_{H}\right)$ for every $g \in G$. Similarly for $X_{G}$.

Definition 5.3. Let $G$ and $H$ be finitely generated. We say that an ME-coupling of $G$ and $H$ is an $\ell^{1}-M E$-coupling or an integrable $M E$-coupling if it admits integrable $G$ - and $H$-fundamental domains. We say that $G$ and $H$ are $\ell^{1}$-measure equivalent if there exists an $\ell^{1}$-ME-coupling of $G$ and $H$.

Remark 5.4. Measure equivalence and $\ell^{1}$-measure equivalence are equivalence relations on countable and finitely generated groups, respectively (see [7], [1]). 
Remark 5.5. A locally compact group $G$ with its Haar measure is an ME-coupling for all its lattices; it is an integrable ME-coupling for every pair of cocompact lattices in $G$.

By [23], Theorem 3.6, the isometry group Isom $\left(\mathbb{H}^{n}\right)$ of the $n$-dimensional hyperbolic space with $n \geq 3$ endowed with its Haar measure is an $\ell^{1}$-ME-coupling for all its lattices. Shalom in loc. cit. was concerned with $\ell^{2}$-integrability and showed that all lattices in simple Lie groups not locally isomorphic to $\operatorname{Isom}\left(\mathbb{H}^{2}\right) \cong \mathrm{PSL}_{2}(\mathbb{R})$ or $\operatorname{Isom}\left(\mathbb{H}^{3}\right) \cong \mathrm{PSL}_{2}(\mathbb{C})$ are $\ell^{2}$-integrable. However, his proof also implies the above statement.

5.2. Bounded cohomology and ME-induction. We briefly recollect basic notions of bounded cohomology.

Let $G$ be a discrete group and $E$ be a real Banach space with isometric $G$-action. We denote by $C_{\mathrm{b}}^{k}(G, E)$ the Banach space $L^{\infty}\left(G^{k+1}, E\right)$ consisting of bounded maps from $G^{k+1}$ to $E$ endowed with the supremum norm and the isometric $G$-action:

$$
(g \cdot f)\left(g_{0}, \ldots, g_{k}\right)=g \cdot f\left(g^{-1} g_{0}, \ldots, g^{-1} g_{k}\right) .
$$

The sequence of Banach $G$-modules $C_{\mathrm{b}}^{k}(G, E), k \geq 0$, becomes a chain complex of Banach $G$-modules via the standard homogeneous coboundary operator

$$
d(f)\left(g_{0}, \ldots, g_{k}\right)=\sum_{i \geq 0}^{k}(-1)^{i} f\left(g_{0}, \ldots, \widehat{g_{i}}, \ldots, g_{k}\right) .
$$

The bounded cohomology $H_{\mathrm{b}}^{*}(G, E)$ of $G$ with coefficients $E$ is the cohomology of the complex of $G$-invariants $C_{\mathrm{b}}^{*}(G, E)^{G}$. The bounded cohomology $H_{\mathrm{b}}^{*}(G, E)$ inherits a semi-norm from $C_{\mathrm{b}}^{*}(G, E)$ : The (semi-)norm of an element $x \in H_{\mathrm{b}}^{k}(G, E)$ is the infimum of the norms of all cocycles in the cohomology class $x$.

The topological dual of the complex of Banach spaces

$$
C_{*}^{(1)}(G) \hat{\otimes}_{\mathbb{Z} G} E \cong C_{*}^{(1)}(G, \mathbb{R}) \hat{\otimes}_{\mathbb{R} G} E,
$$

whose homology $H_{*}^{(1)}(G, E)$ is the so-called $\ell^{1}$-homology of $G$ with coefficients $E$ (compare Remark 2.11), is canonically isomorphic to $C_{\mathrm{b}}^{*}\left(G, E^{\prime}\right)$ (see [20], Proposition 2.3.1 on p. 20). Thus, we obtain a natural pairing, which descends to (co-) homology (both pairings are denoted by $\left\langle_{-},{ }_{-}\right\rangle$):

$$
\left\langle{ }_{-}{ }_{-}\right\rangle: H_{\mathrm{b}}^{k}\left(G, E^{\prime}\right) \otimes H_{k}^{(1)}(G, E) \rightarrow \mathbb{R} .
$$

In the next theorem we identify the set $H x \cap X_{H}$ which consists of just one element with the element itself.

Theorem 5.6 (Monod-Shalom). Let $(\Omega, \mu)$ be an ME-coupling of $G$ and $H$. Let $X_{G}$ and $X_{H}$ be measurable fundamental domains for the $G$ - and $H$-action on $\Omega$, 
respectively. Let $\alpha: H \times X_{G} \rightarrow G$ be the cocycle associated to $X_{G}$. The maps

$$
\begin{gathered}
\alpha^{*}: C_{\mathrm{b}}^{*}\left(G, L^{\infty}\left(X_{H}, \mathbb{R}\right)\right) \rightarrow C_{\mathrm{b}}^{*}\left(H, L^{\infty}\left(X_{G}, \mathbb{R}\right)\right) \\
\alpha^{k} f\left(h_{0}, \ldots, h_{k}\right)(x)=f\left(\alpha\left(h_{0}^{-1}, x\right)^{-1}, \ldots, \alpha\left(h_{k}^{-1}, x\right)^{-1}\right)\left(H x \cap X_{H}\right)
\end{gathered}
$$

define a chain map that restricts to the invariants

$$
\alpha^{*}: C_{\mathrm{b}}^{*}\left(G, L^{\infty}\left(X_{H}, \mathbb{R}\right)\right)^{G} \rightarrow C_{\mathrm{b}}^{*}\left(H, L^{\infty}\left(X_{G}, \mathbb{R}\right)\right)^{H}
$$

and induces an isometric isomorphism

$$
H_{\mathrm{b}}^{*}(\alpha): H_{\mathrm{b}}^{*}\left(G, L^{\infty}\left(X_{H}, \mathbb{R}\right)\right) \stackrel{\cong}{\longrightarrow} H_{\mathrm{b}}^{*}\left(H, L^{\infty}\left(X_{G}, \mathbb{R}\right)\right) .
$$

in cohomology. The map $H_{\mathrm{b}}^{*}(\Omega)$ given by the commutative diagram

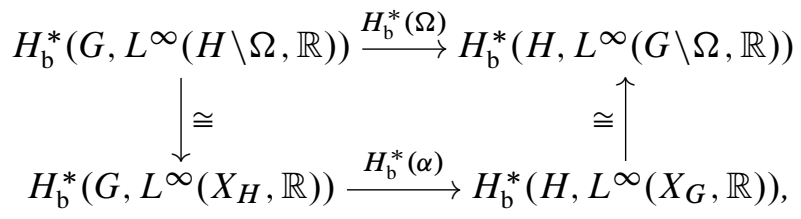

where the vertical isomorphisms are induced by the (restrictions) of the projections $X_{H} \rightarrow H \backslash \Omega$ and $X_{G} \rightarrow G \backslash \Omega$, respectively, does not depend on the choices of fundamental domains.

Proof. Apart from the fact that the isomorphism is isometric, this is exactly Proposition 4.6 in [21] (with $S=\Omega$ and $E=\mathbb{R}$ ). The proof therein relies on [20], Theorem 7.5.3 in $\S 7$, which also yields the isometry statement.

To formulate the next theorem, consider the measurable and countable-to-one map

$$
\begin{gathered}
\phi_{k}^{\alpha}: H^{k+1} \times X_{G} \rightarrow G^{k+1} \times X_{H}, \\
\left(h_{0}, \ldots, h_{k}, x\right) \mapsto\left(\alpha\left(h_{0}^{-1}, x\right)^{-1}, \ldots, \alpha\left(h_{k}^{-1}, x\right)^{-1}, H x \cap X_{H}\right) .
\end{gathered}
$$

Theorem 5.7. Retain the notation of Theorem 5.6. Let $c_{\Omega}=\mu\left(X_{H}\right) / \mu\left(X_{G}\right)$ be the coupling index. We equip $X_{G}$ and $X_{H}$ with the normalized measures $\left.\mu\left(X_{G}\right)^{-1} \mu\right|_{X_{G}}$ and $\left.\mu\left(X_{H}\right)^{-1} \mu\right|_{X_{H}}$. Then

$$
\begin{gathered}
\alpha_{k}: C_{k}^{(1)}(H) \hat{\otimes}_{\mathbb{Z}} L^{1}\left(X_{G}, \mathbb{R}\right) \rightarrow C_{k}^{(1)}(G) \hat{\otimes}_{\mathbb{Z}} L^{1}\left(X_{H}, \mathbb{R}\right), \\
\alpha_{k} f(\bar{g}, x)=c_{\Omega} \cdot \sum_{(\bar{h}, y) \in\left(\phi_{k}^{\alpha}\right)^{-1}(\bar{g}, x)} f(\bar{h}, y)
\end{gathered}
$$

defines, using the identification in Example 2.10, a chain map that descends to the coinvariants

$$
C_{*}^{(1)}(H) \hat{\otimes}_{\mathbb{Z} H} L^{1}\left(X_{G}, \mathbb{R}\right) \rightarrow C_{*}^{(1)}(G) \hat{\otimes}_{\mathbb{Z} G} L^{1}\left(X_{H}, \mathbb{R}\right)
$$


and induces an isometric isomorphism

$$
H_{*}^{(1)}(\alpha): H_{*}^{(1)}\left(H, L^{1}\left(X_{G}, \mathbb{R}\right)\right) \rightarrow H_{*}^{(1)}\left(G, L^{1}\left(X_{H}, \mathbb{R}\right)\right) .
$$

Furthermore, the dual of the map (5.2) is the map (5.1).

We need the following general (and easy) lemma

Lemma 5.8. Let $\left(X, v_{X}\right)$ and $\left(Y, v_{Y}\right)$ be standard measure spaces and $p: X \rightarrow Y$ a measurable map such that

(1) the fiber $p^{-1}(y)$ is countable for $v_{Y}$-a.e. $y \in Y$, and

(2) $p$ is locally measure-preserving, that is, if $\left.p\right|_{A}$ is injective for a measurable $A \subset X$, then $v_{X}(A)=v_{Y}(p(A))$.

Then for any $f \in L^{1}\left(X, v_{X}\right)$ the function $y \mapsto \sum_{x \in p^{-1}(y)} f(x)$ is $v_{Y}$-integrable and

$$
\int_{X} f d v_{X}=\int_{Y} \sum_{x \in p^{-1}(y)} f(x) d v_{Y}(y) .
$$

Proof. The assertion is obvious for $f=\chi_{A}$ being the characteristic function of a measurable subset $A \subset X$ for which $\left.p\right|_{A}$ is injective. By the selection theorem every $f \in L^{1}\left(X, v_{X}\right)$ can be approximated by linear combinations of such characteristic functions which proves the lemma.

Proof of Theorem 5.7. We verify that the dual of map (5.2) is the map (5.1), that is, for $f \in C_{k}^{(1)}(H) \hat{\otimes}_{\mathbb{Z} H} L^{1}\left(X_{G}, \mathbb{R}\right)$ and $g \in C_{\mathrm{b}}^{k}\left(G, L^{\infty}\left(X_{H}, \mathbb{R}\right)\right)$,

$$
\left\langle\alpha_{k} f, g\right\rangle=\left\langle f, \alpha^{k} g\right\rangle
$$

holds true. Since $\phi_{k}^{\alpha}$ is countable-to-one and locally measure-preserving, (5.3) is implied by the previous lemma as follows:

$$
\begin{aligned}
\left\langle\alpha_{k} f, g\right\rangle & =\frac{\mu\left(X_{H}\right)}{\mu\left(X_{G}\right)} \sum_{\bar{g} \in G^{k+1}} \int_{X_{H}} \sum_{\substack{(\bar{h}, y) \in H^{k+1} \times X_{G} \\
\phi_{k}^{\alpha}(\bar{h}, y)=(\bar{g}, x)}} f(\bar{h}, y) g(\bar{g}, x) \mu\left(X_{H}\right)^{-1} d \mu(x) \\
& =\mu\left(X_{G}\right)^{-1} \sum_{\bar{g} \in G^{k+1}} \int_{X_{H}} \sum_{\substack{(\bar{h}, y) \in H^{k+1} \times X_{G} \\
\phi_{k}^{\alpha}(\bar{h}, y)=(\bar{g}, x)}} f(\bar{h}, y) g \circ \phi_{k}^{\alpha}(\bar{h}, y) d \mu(x) \\
& =\mu\left(X_{G}\right)^{-1} \cdot \sum_{\bar{h} \in H^{k+1}} \int_{X_{G}} f(\bar{h}, x) g \circ \phi_{k}^{\alpha}(\bar{h}, x) d \mu(x) \\
& =\left\langle f, \alpha^{k} g\right\rangle .
\end{aligned}
$$


Since $\alpha^{*}$ is a chain map, we know that the dual of $\alpha_{k} d-d \alpha_{k+1}$ vanishes. The Hahn-Banach theorem implies that $\alpha_{k} d-d \alpha_{k+1}=0$, so $\alpha_{*}$ is also a chain map. Similarly one concludes that $\alpha_{*}$ descends to the coinvariants from the fact that $\alpha^{*}$ restricts to the invariants.

Since $H_{\mathrm{b}}^{k}(\alpha)$ is an isometric isomorphism, also $H_{k}^{(1)}(\alpha)$ is an isometric isomorphism by [15], Theorem 1.1.

Remark 5.9. With more effort one can also show that $H_{*}^{(1)}(\alpha)$ does not depend on the choice of the fundamental domains, thus could be rightfully denoted by $H_{*}^{(1)}(\Omega)$ similar to the cohomological case. Since we do not need this, we refrain from proving this.

\subsection{Invariance of the simplicial volume}

Lemma 5.10. Let $(\Omega, \mu)$ be an $M E$-coupling of two finitely generated groups $G$ and $H$. Let $X_{G}$ and $X_{H}$ be fundamental domains of the $G$ - and $H$-action, respectively. Let $c_{\Omega}=\mu\left(X_{H}\right) / \mu\left(X_{G}\right)$ be the coupling index. Assume that $X_{G}$ is integrable, and let $\alpha: H \times X_{G} \rightarrow G$ be the associated integrable cocycle. Then the image of the composition

$$
H_{*}(H, \mathbb{Z}) \rightarrow H_{*}^{(1)}\left(H, L^{1}\left(X_{G}, \mathbb{R}\right)\right) \stackrel{H_{*}^{(1)}(\alpha)}{\longrightarrow} H_{*}^{(1)}\left(G, L^{1}\left(X_{H}, \mathbb{R}\right)\right)
$$

is contained in

$$
c_{\Omega} \cdot \operatorname{im}\left(H_{*}^{(1,1)}\left(G, L^{1}\left(X_{H}, \mathbb{Z}\right)\right) \rightarrow H_{*}^{(1)}\left(G, L^{1}\left(X_{H}, \mathbb{R}\right)\right)\right),
$$

where all maps except $H_{*}^{(1)}(\alpha)$ are the composition of the corresponding comparison and coefficient change maps.

Proof. For a $(k+1)$-tuple $\bar{h}=\left(h_{0}, \ldots, h_{k}\right) \in H^{k+1}$ we abbreviate

$$
\begin{gathered}
\alpha(\bar{h}, x)=\left(\alpha\left(h_{0}, x\right), \ldots, \alpha\left(h_{k}, x\right)\right), \\
\bar{h}^{-1}=\left(h_{0}^{-1}, \ldots, h_{k}^{-1}\right) .
\end{gathered}
$$

We use the identifications in Example 2.10. The image of $C_{k}(H)=C_{k}(H) \otimes_{\mathbb{Z}} \mathbb{Z}$ in

$$
C_{k}^{(1)}(H) \hat{\otimes}_{\mathbb{Z}} L^{1}\left(X_{G}, \mathbb{Z}\right) \cong L^{1}\left(H^{k+1} \times X_{G}, \mathbb{Z}\right)
$$

is certainly contained in the set of bounded measurable functions $f: H^{k+1} \times X_{G} \rightarrow$ $\mathbb{Z}$ for which there is a finite subset $F \subset H^{k+1}$ such that $f$ is supported on $F \times X_{G}$. Let $f: H^{k+1} \times X_{G} \rightarrow \mathbb{Z}$ be such. It is immediate from (5.2) that $c_{\Omega}^{-1} \cdot \alpha_{k} f$ is $\mathbb{Z}$-valued. So it remains to show that

$$
\int_{G^{k+1} \times X_{H}}\left|\alpha_{k} f(\bar{g}, y)\right| \operatorname{diam}(\bar{g})<\infty .
$$


Using Lemma 5.8 this is implied by

$$
\begin{aligned}
& \int_{G^{k+1} \times X_{H}}\left|\alpha_{k} f(\bar{g}, y)\right| \operatorname{diam}(\bar{g}) \\
& \quad \leq \int_{G^{k+1} \times X_{H}} \int_{\left(\phi_{k}^{\alpha}\right)^{-1}(\bar{g}, y)}|f(\bar{h}, x)| \operatorname{diam}(\bar{g}) \\
& \quad=\int_{G^{k+1} \times X_{H}} \int_{\left(\phi_{k}^{\alpha}\right)^{-1}(\bar{g}, y)}|f(\bar{h}, x)| \operatorname{diam}\left(\bar{\alpha}\left(\bar{h}^{-1}, x\right)^{-1}\right) \\
& \quad \stackrel{5.8}{=} \int_{H^{k+1} \times X_{G}}|f(\bar{h}, x)| \operatorname{diam}\left(\bar{\alpha}\left(\bar{h}^{-1}, x\right)^{-1}\right) \\
& \leq \operatorname{ess-sup}(f) \cdot \int_{H^{k+1} \times X_{G}} \operatorname{diam}\left(\bar{\alpha}\left(\bar{h}^{-1}, x\right)^{-1}\right)<\infty .
\end{aligned}
$$

The last step follows from the integrability.

Let $N$ be an aspherical topological space, and let $H=\pi_{1}(N)$. By asphericity and the fundamental lemma of homological algebra there is up to equivariant chain homotopy a unique $H$-equivariant chain homotopy equivalence

$$
c_{H}: C_{*}(\tilde{N}) \rightarrow C_{*}(H)
$$

from the singular chain complex of the universal cover $\tilde{N}$ to the standard resolution of $H$. By a theorem of Gromov [14], Theorem 4.1, the map $c_{H}$ induces an isometric isomorphism in bounded cohomology with $\mathbb{R}$-coefficients. By the translation principle in [15], Theorem 1.1, $c_{H}$ induces an isometric isomorphism in $\ell^{1}$-homology, and thus (compare [15], Proposition 2.4) the induced map in homology is an isometric isomorphism:

Lemma 5.11. Let $N$ be aspherical and $H=\pi_{1}(N)$. The canonical map

$$
H_{*}\left(c_{H}\right): H_{*}(N, \mathbb{R}) \rightarrow H_{*}(H, \mathbb{R})
$$

is an isometric isomorphism with respect to the semi-norms induced by the $\ell^{1}$-norms.

Lemma 5.12. The simplicial volume of an aspherical, orientable, closed manifold with word-hyperbolic fundamental group and of dimension at least 2 does not vanish.

Proof. Let $N$ be such a manifold. Since the comparison map from the bounded cohomology of $H=\pi_{1}(N)$ to the cohomology of $H$, which is isomorphic to the one of $N$ by asphericity, is surjective for $\mathbb{R}$-coefficients by [19], Theorem 3, the cohomological fundamental class of $N$ has a bounded representative. Thus the simplicial volume of $N$ is positive [10], Corollary in Section 1.1. 
Theorem 5.13. Let $M$ and $N$ be closed, oriented manifolds of dimension $n \geq 2$ whose fundamental groups $G=\pi_{1}(M)$ and $H=\pi_{1}(N)$ are word-hyperbolic. Let $(\Omega, \mu)$ be an ergodic, integrable ME-coupling $(\Omega, \mu)$ of $G$ and $H$ with coupling index $c=\mu(H \backslash \Omega) / \mu(G \backslash \Omega)$.

Let $x_{G} \in H^{n}(G, \mathbb{R})$ be the element that maps to the cohomological fundamental class of $M$ under the isomorphism $H^{n}\left(c_{G}\right): H^{n}(G) \rightarrow H^{n}(M)$. We define $x_{H} \in$ $H^{n}(H, \mathbb{R})$ analogously.

Suppose that $x_{G}^{\mathrm{b}} \in H_{\mathrm{b}}^{n}(G, \mathbb{R})$ is an element that maps to $x_{G}$ under the comparison (forgetful) map $H_{\mathrm{b}}^{n}(G, \mathbb{R}) \rightarrow H^{n}(G, \mathbb{R})$. Consider the composition

$$
\begin{aligned}
& H_{\mathrm{b}}^{n}(G, \mathbb{R}) \rightarrow H_{\mathrm{b}}^{n}\left(G, L^{\infty}(H \backslash \Omega, \mathbb{R})\right) \\
& \quad \stackrel{H_{\mathrm{b}}^{n}(\Omega)}{\longrightarrow} H_{\mathrm{b}}^{n}\left(H, L^{\infty}(G \backslash \Omega, \mathbb{R})\right) \stackrel{I^{n}}{\longrightarrow} H_{\mathrm{b}}^{n}(H, \mathbb{R}) \rightarrow H^{n}(H, \mathbb{R})
\end{aligned}
$$

where the first map is induced by the inclusion of constant functions, $I^{n}$ is the map induced by integration in the coefficients and the last map is the comparison map. Then $x_{G}^{\mathrm{b}}$ is mapped to $\pm c \cdot x_{H}$ under (5.6).

Proofs of Theorems 1.9 and 5.13. Let $H_{n}\left(i_{H}\right): H_{n}(H, \mathbb{R}) \rightarrow H_{n}^{(1)}(H, \mathbb{R})$ denote the comparison map; it is isometric with respect to the semi-norms induced by the $\ell^{1}$-norm on the chain complexes: This follows from the fact that $C_{*}(H) \otimes_{\mathbb{Z} H} \mathbb{R} \rightarrow$ $C_{*}^{(1)}(H) \hat{\otimes}_{\mathbb{Z} H} \mathbb{R}$ is isometric and has dense image (compare [15], Proposition 2.4). We denote - by a slight abuse of notation - the comparison (forgetful) map for the group $H$ in bounded cohomology by $H^{n}\left(i_{H}\right): H_{\mathrm{b}}^{n}(H, \mathbb{R}) \rightarrow H^{n}(H, \mathbb{R})$. We define $H_{n}\left(i_{G}\right)$ and $H^{n}\left(i_{G}\right)$ for the group $G$ similarly.

Let $X_{H} \subset \Omega$ and $X_{G} \subset \Omega$ be integrable fundamental domains of the $H$-action and $G$-action on $\Omega$, respectively. Let $\alpha: H \times X_{G} \rightarrow G$ be the cocycle associated to $X_{G}$. For the following we endow $X_{H}$ and $X_{G}$ with the normalized measures $\left.\mu\left(X_{H}\right)^{-1} \mu\right|_{X_{H}}$ and $\left.\mu\left(X_{G}\right)^{-1} \mu\right|_{X_{G}}$, respectively.

With normalization, the chain map $j_{H}: C_{*}^{(1)}(H) \hat{\otimes}_{\mathbb{Z} H} \mathbb{R} \rightarrow C_{*}^{(1)}(H) \hat{\otimes}_{\mathbb{Z} H}$ $L^{1}\left(X_{G}, \mathbb{R}\right)$ given by the inclusion of constant functions is isometric. Integration in $L^{1}\left(X_{G}, \mathbb{R}\right)$ provides a norm-decreasing left inverse. Hence the induced map in $\ell^{1}$-homology

$$
H_{n}^{(1)}\left(j_{H}\right): H_{n}^{(1)}(H, \mathbb{R}) \rightarrow H_{n}^{(1)}\left(H, L^{1}\left(X_{G}, \mathbb{R}\right)\right)
$$

is isometric. Again by a slight abuse of notation, we denote the map in bounded cohomology induced by inclusion of constants maps by

$$
H_{\mathrm{b}}^{n}\left(j_{H}\right): H_{\mathrm{b}}^{n}(H, \mathbb{R}) \rightarrow H_{\mathrm{b}}^{n}\left(H, L^{\infty}\left(X_{G}, \mathbb{R}\right)\right) .
$$

We define the map $j_{G}$ for the group $G$ similarly.

We start with the proof of Theorem 1.9. Let $m=\operatorname{dim}(M)$ and $n=\operatorname{dim}(N)$. Assume that $\|N\|>0$. Let $[N] \in H_{n}(N, \mathbb{R})$ be the homological fundamental class 
of $N$. Since each map in the composition

$$
\begin{aligned}
& H_{n}(H, \mathbb{R}) \stackrel{H_{n}\left(i_{H}\right)}{\longrightarrow} H_{n}^{(1)}(H, \mathbb{R}) \\
& \quad \stackrel{H_{n}^{(1)}\left(j_{H}\right)}{\longrightarrow} H_{n}^{(1)}\left(H, L^{1}\left(X_{G}, \mathbb{R}\right)\right) \stackrel{H_{n}^{(1)}(\alpha)}{\longrightarrow} H_{n}^{(1)}\left(G, L^{1}\left(X_{H}, \mathbb{R}\right)\right)
\end{aligned}
$$

is isometric with respect to the semi-norms induced by the respective $\ell^{1}$-norms (see Theorem 5.7) and $H_{n}(H, \mathbb{R})$ is generated by the element $H_{n}\left(c_{H}\right)([N])$ with positive semi-norm (Lemma 5.11), we obtain that $H_{n}^{(1)}(\alpha) \circ H_{n}^{(1)}\left(j_{H}\right) \circ H_{n}\left(i_{H}\right)$ is injective. Lemma 5.10 and the fact that $[N] \in \operatorname{im}\left(H_{n}(N, \mathbb{Z}) \rightarrow H_{n}(N, \mathbb{R})\right)$ yield that

$$
\begin{aligned}
& 0 \neq H_{n}^{(1)}(\alpha) \circ H_{n}^{(1)}\left(j_{H}\right) \circ H_{n}\left(i_{H}\right) \circ H_{n}\left(c_{H}\right)([N]) \\
& \quad \in c_{\Omega} \cdot \operatorname{im}\left(H_{n}^{(1,1)}\left(G, L^{1}\left(X_{H}, \mathbb{Z}\right)\right) \rightarrow H_{n}^{(1)}\left(G, L^{1}\left(X_{H}, \mathbb{R}\right)\right)\right) .
\end{aligned}
$$

If $G$ is word-hyperbolic, then Theorem 1.5 implies that

$$
\begin{aligned}
& H_{n}^{(1)}(\alpha) \circ H_{n}^{(1)}\left(j_{H}\right) \circ H_{n}\left(i_{H}\right) \circ H_{n}\left(c_{H}\right)([N]) \\
& \quad \in c_{\Omega} \cdot \operatorname{im}\left(H_{n}\left(G, L^{1}\left(X_{H}, \mathbb{Z}\right)\right) \rightarrow H_{n}^{(1)}\left(G, L^{1}\left(X_{H}, \mathbb{R}\right)\right)\right) .
\end{aligned}
$$

In particular, $H_{n}\left(G, L^{1}\left(X_{H}, \mathbb{Z}\right)\right) \neq 0$, which implies that $n \leq m=\operatorname{dim}(M)$.

Next assume that $H$ and $G$ are both word-hyperbolic. By Lemma 5.12 $M$ and $N$ have positive simplicial volume. From the argument above and by symmetry we conclude that $m=n$.

The group $G$ is an orientable Poincare duality group; the Poincare duality isomorphism is functorial with respect to coefficient homomorphisms. Further, for any coefficient module $E$ there is a functorial isomorphism $H^{0}(G, E) \cong E^{G}$. Thus we obtain a commutative diagram:

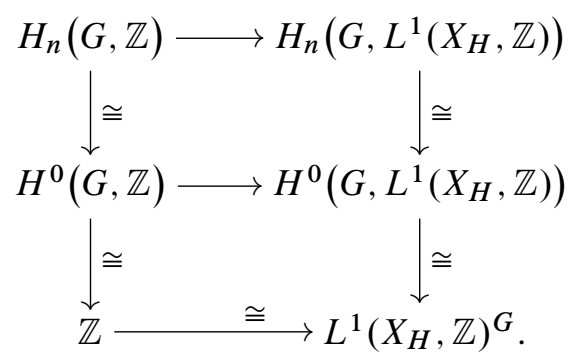

The bottom map is an isomorphism because of ergodicity. In combination with (5.7) this implies that there is a non-zero integer $e \in \mathbb{Z}$ such that

$$
\begin{aligned}
& H_{n}^{(1)}(\alpha) \circ H_{n}^{(1)}\left(j_{H}\right) \circ H_{n}\left(i_{H}\right) \circ H_{n}\left(c_{H}\right)([N]) \\
& \quad=e \cdot c_{\Omega} \cdot H_{n}^{(1)}\left(j_{G}\right) \circ H_{n}\left(i_{G}\right) \circ H_{n}\left(c_{G}\right)([M]) .
\end{aligned}
$$

Since the maps involved here are isometric and $|e| \geq 1$, this implies that

$$
\|N\| \geq c_{\Omega} \cdot\|M\| \text {. }
$$


By interchanging the roles of $H$ and $G$, we obtain similarly $\|M\| \geq c^{-1} \cdot\|N\|$ and thus

$$
e= \pm 1 \quad \text { and } \quad\|M\|=c_{\Omega} \cdot\|N\|,
$$

which concludes the proof of Theorem 1.9.

Next we prove Theorem 5.13. The assumptions imply that $M$ and $N$ have positive simplicial volume (see Lemma 5.12). Hence we know from the argument above that (5.8) holds with $e= \pm 1$. The assertion follows from the fact that $H^{n}(H, \mathbb{R}) \cong \mathbb{R}$ and $H^{n}\left(i_{H}\right) \circ I^{n} \circ H_{\mathrm{b}}^{n}(\Omega) \circ H_{\mathrm{b}}^{n}\left(j_{G}\right)\left(x_{G}^{\mathrm{b}}\right)$ evaluated against the image $H_{n}\left(\left[c_{H}\right]\right)([N])$ of the fundamental class of $[N]$ is $\pm c_{\Omega}$ :

$$
\begin{aligned}
\left\langle H^{n}\left(i_{H}\right) \circ\right. & \left.I^{n} \circ H_{\mathrm{b}}^{n}(\Omega) \circ H_{\mathrm{b}}^{n}\left(j_{G}\right)\left(x_{G}^{\mathrm{b}}\right), H_{n}\left(c_{H}\right)([N])\right\rangle \\
& =\left\langle H^{n}\left(i_{H}\right) \circ I^{n} \circ H_{\mathrm{b}}^{n}(\alpha) \circ H_{\mathrm{b}}^{n}\left(j_{G}\right)\left(x_{G}^{\mathrm{b}}\right), H_{n}\left(c_{H}\right)([N])\right\rangle \\
& =\left\langle I^{n} \circ H_{\mathrm{b}}^{n}(\alpha) \circ H_{\mathrm{b}}^{n}\left(j_{G}\right)\left(x_{G}^{\mathrm{b}}\right), H_{n}\left(i_{H}\right) \circ H_{n}\left(c_{H}\right)([N])\right\rangle \\
& =\left\langle H_{\mathrm{b}}^{n}(\alpha) \circ H_{\mathrm{b}}^{n}\left(j_{G}\right)\left(x_{G}^{\mathrm{b}}\right), H_{n}^{(1)}\left(j_{H}\right) \circ H_{n}\left(i_{H}\right) \circ H_{n}\left(c_{H}\right)([N])\right\rangle \\
& =\left\langle H_{\mathrm{b}}^{n}\left(j_{G}\right)\left(x_{G}^{\mathrm{b}}\right), H_{n}^{(1)}(\alpha) \circ H_{n}^{(1)}\left(j_{H}\right) \circ H_{n}\left(i_{H}\right) \circ H_{n}\left(c_{H}\right)([N])\right\rangle \\
& \stackrel{(5.8)}{=} \pm c_{\Omega} \cdot\left\langle H_{\mathrm{b}}^{n}\left(j_{G}\right)\left(x_{G}^{\mathrm{b}}\right), H_{n}^{(1)}\left(j_{G}\right) \circ H_{n}\left(i_{G}\right) \circ H_{n}\left(c_{G}\right)([M])\right\rangle \\
& = \pm c_{\Omega} \cdot\left\langle x_{G}^{\mathrm{b}}, H_{n}\left(i_{G}\right) \circ H_{n}\left(c_{G}\right)([M])\right\rangle \\
& = \pm c_{\Omega} \cdot\left\langle x_{G}, H_{n}\left(c_{G}\right)([M])\right. \\
& = \pm c_{\Omega} .
\end{aligned}
$$

\section{References}

[1] U. Bader, A. Furman, and R. Sauer, Integrable measure equivalence and rigidity of hyperbolic lattices. Invent. Math., to appear. Doi 10.1007/s00222-012-0445-9

[2] M. R. Bridson and A. Haefliger, Metric spaces of non-positive curvature. Grundlehren Math. Wiss. 319, Springer-Verlag, Berlin 1999. Zbl 0988.53001 MR 1744486

[3] M. Burger, A. Iozzi, N. Monod, and A. Wienhard, Bounds for cohomology classes. Enseign. Math. (2) 54 (2008), 52-54.

[4] J. B. Conway, A course in functional analysis. 2nd ed., Grad. Texts in Math. 96, SpringerVerlag, New York 1990. Zbl 0706.46003 MR 1070713

[5] M. Coornaert, T. Delzant, and A. Papadopoulos, Géométrie et théorie des groupes. Lecture Notes in Math. 1441, Springer-Verlag, Berlin 1990. Zbl 0727.20018 MR 1075994

[6] J. Diestel and J. J. Uhl, Jr., Vector measures. Amer. Math. Soc., Providence, R.I., 1977. Zbl 0369.46039 MR 0453964

[7] A. Furman, Gromov's measure equivalence and rigidity of higher rank lattices. Ann. of Math. (2) 150 (1999), 1059-1081. Zbl 0943.22013 MR 1740986

[8] A. Furman, A survey of measured group theory. In Geometry, rigidity, and group actions, Chicago Lectures in Math., University of Chicago Press, Chicago 2011, 296--374. Zbl 06077151 MR 2807836 
[9] D. Gaboriau, Invariants $l^{2}$ de relations d'équivalence et de groupes. Publ. Math. Inst. Hautes Études Sci. 95 (2002), 93-150. Zbl 1022.37002 MR 1953191

[10] M. Gromov, Volume and bounded cohomology. Inst. Hautes Études Sci. Publ. Math. 56 (1982), 5-99. Zbl 0516.53046 MR 686042 (84h:53053)

[11] M. Gromov, Hyperbolic groups. In Essays in group theory, Math. Sci. Res. Inst. Publ. 8, Springer-Verlag, New York 1987, 75-263. Zbl 0634.20015 MR 0919829

[12] M. Gromov, Geometric group theory (Sussex, 1991), vol. 2: Asymptotic invariants of infinite groups. London Math. Soc. Lecture Note Ser. 182, Cambridge University Press, Cambridge 1993. Zbl 0841.20039 MR 1253544

[13] A. Grothendieck, Produits tensoriels topologiques et espaces nucléaires. Mem. Amer. Math. Soc. 161955 (1955), no. 16. Zbl 0064.35501 MR 0075539

[14] N. V. Ivanov, Foundations of the theory of bounded cohomology. Zap. Nauchn. Sem. Leningrad. Otdel. Mat. Inst. Steklov. (LOMI) 143 (1985), 69-109; English transl. J. Math. Sci. 37 (1987), 1090-1115. Zbl 0612.55006 MR 806562

[15] C. Löh, Isomorphisms in $\ell^{1}$-homology. Münster J. Math. 1 (2008), 237-265. Zbl 1158.55007 MR 2502500

[16] W. Lück, $L^{2}$-invariants: theory and applications to geometry and $K$-theory. Ergeb. Math. Grenzgeb. (3) 44, Springer-Verlag, Berlin 2002. Zbl 1009.55001 MR 1926649

[17] S. Matsumoto and S. Morita, Bounded cohomology of certain groups of homeomorphisms. Proc. Amer. Math. Soc. 94 (1985), 539-544. Zbl 0536.57023 MR 787909

[18] I. Mineyev, Higher dimensional isoperimetric functions in hyperbolic groups. Math. Z. 233 (2000), 327-345. Zbl 0954.20021 MR 1743440

[19] I. Mineyev, Bounded cohomology characterizes hyperbolic groups. Q. J. Math. 53 (2002), 59-73. Zbl 1013.20048 MR 1887670

[20] N. Monod, Continuous bounded cohomology of locally compact groups. Lecture Notes in Math. 1758, Springer-Verlag, Berlin 2001. Zbl 0967.22006 MR 1840942

[21] N. Monod and Y. Shalom, Orbit equivalence rigidity and bounded cohomology. Ann. of Math. (2) 164 (2006), 825-878. Zbl 1129.37003 MR 2259246

[22] S. Popa, Deformation and rigidity for group actions and von Neumann algebras. In Proc. Internat. Congr. Mathematicians, Vol. I, Eur. Math. Soc., Zürich 2007, 445-477. Zbl 1132.46038 MR 2334200

[23] Y. Shalom, Rigidity, unitary representations of semisimple groups, and fundamental groups of manifolds with rank one transformation group. Ann. of Math. (2) 152 (2000), 113-182. Zbl 0970.22011 MR 1792293

[24] Y. Shalom, Measurable group theory. In European Congress of Mathematics, Eur. Math. Soc., Zürich 2005, 391-423. Zbl 1137.37301 MR 2185757

Received May 17, 2010; revised September 3, 2011

U. Bader, Mathematics Department, Technion - Israel Institute of Technology, Haifa, 32000, Israel

E-mail: bader@tx.technion.ac.il 
A. Furman, Department of Mathematics, Statistics, and Computer Science, University of Illinois at Chicago, 322 Science and Engineering Offices (M/C 249), 851 S. Morgan Street, Chicago, IL 60607-7045, U.S.A.

E-mail: furman@math.uic.edu

R. Sauer, Department of Mathematics, Karlsruhe Institute of Technology, 76128 Karlsruhe, Germany

E-mail: roman.sauer@kit.edu 